\title{
Clutch size in populations and species of cnemidophorines (Squamata: Teiidae) on the eastern coast of Brazil
}

\author{
VANDERLAINE A. MENEZES ${ }^{1}$ and CARLOS F.D. ROCHA ${ }^{1}$ \\ ${ }^{1}$ Laboratório de Vertebrados, Departamento de Ecologia, Universidade do Estado do Rio de Janeiro, \\ Rua São Francisco Xavier, 524, PHLC, Sala 220, Maracanã, 20550-013 Rio de Janeiro, RJ, Brasil \\ Manuscript received on September 6, 2012; accepted for publication on May 8, 2013
}

\begin{abstract}
We analyzed some reproductive aspects of 16 coastal populations, belonging to five lizard species (A. ocellifera, A. abaetensis, A. nativo, A. littoralis and C. lacertoides) from different restinga habitats along the eastern coast of Brazil. This study aimed to evaluate to what extent the reproductive aspects vary geographically and among species. For each female, we recorded the number of vitellogenic follicles, size and color of the largest follicle, presence and size of corpora lutea, and number and size of oviductal eggs. Clutch size of almost all coastal populations/species of Ameivula had little variation and most clutches were composed of two eggs. There was a significant relationship between female size and the mean clutch size when females from different species were pooled. Mean egg volume, among species, varied from 420 to $655 \mathrm{~mm}^{3}$. Relative clutch mass varied from 0.129 to 0.159 and did not differ significantly among species. We concluded that the five coastal species studied (four bisexuals and one parthenogenetic) had similar reproductive characteristics. Most of them presented multiple clutches, low clutch size and low relative clutch mass, similar to other species in the genus and to unisexual and bisexual species of the Teiidae family.
\end{abstract}

Key words: clutch size, Ameivula, reproduction, restinga.

\section{INTRODUCTION}

Differences in lizard reproduction traits such as clutch size, growth rates and adult sizes occur among species and populations within a species (e.g. Kiefer et al. 2008, Galdino and Van Sluys 2011). Such variations can occur in response to different local environmental conditions (e.g. Shine and Elphick 2001, Brandt and Navas 2011), genetic differences among populations/species (e.g. Niewiarowski 1994) and also from evolutionary processes (e.g. Shine and Geer 1991). In Brazil, there are still few

Correspondence to: Vanderlaine Amaral Menezes

E-mail: va.menezes@gmail.com studies that focus on the reproductive traits among different populations within a lizard species (e.g. Kiefer et al. 2008, Galdino and Van Sluys 2011). This lack of knowledge limits our understanding of how various attributes influence the reproduction traits of the populations within a species along its geographical range. So, the changing profiles on the reproductive traits of lizards and the effects of the environment on them need to be better understood.

The reproductive patterns of some cnemidophorines species in tropical areas have been an object of interest in the last two decades, but this knowledge is still limited for many species 
and populations (e.g. Vitt et al. 1997, Mesquita and Colli 2003a, Menezes et al. 2004, Balestrin et al. 2010, Santana et al. 2010). In South America, parthenogenesis in cnemidophorines is known for the Cnemidophorus lemniscatus complex in the Amazon Forest (Vanzolini 1970, Cole and Dessauer 1993) and for Ameivula nativo in eastern Brazil (Rocha et al. 1997, Menezes et al. 2004). Ameivula nativo was the first example of a parthenogenetic species of cnemidophorines found south of the Amazon rainforest (Rocha et al. 1997, 1999). The analysis of reproductive aspects in these cnemidophorines species may increase the understanding of how they interact with the environment in which they live in and allow for comparisons of ecological and physiological aspects of unisexual and bisexual species.

The clutch size is variable in cnemidophorines, with records of species with clutches of a single egg (as in C. arubensis - Vitt and Breitenbach 1993 and C. jalapensis - Colli et al. 2009) up to broods with several eggs (2 to 5 eggs in $C$. lemniscatus - Léon and Cova 1973). Different patterns of the ecology and life history of lizards can affect the clutch size of a given species. The body size of females is among the most obvious factors that can affect the size of clutch or the brood produced (e.g. Vrcibradic and Rocha 2011, Winck and Rocha 2012). Selective forces may either favor a larger clutch size, where each egg has a relatively small volume or, alternatively, may promote the production of a smaller clutch size, in which each egg has a relatively large volume (Lack 1954 apud Sinervo et al. 1992, Kiefer et al. 2008).

Along the areas of restinga in the eastern coast of Brazil four species of Ameivula and one of Contomastix are known to occur: $C$. lacertoides (in southern Brazil), A. littoralis (endemic to the state of Rio de Janeiro), A. nativo (in the states of Espírito Santo and Bahia), A. abaetensis (in the states of Bahia and Sergipe) and $A$. ocellifera (widely distributed in northeastern Brazil) (Menezes and Rocha 2013). Although many studies have contributed to increase the knowledge on reproduction of lizards of these genera (e.g. Léon and Cova 1973, Schall 1983, Vitt 1983, Magnusson 1987, Vitt et al. 1997, Mesquita and Colli 2003a, b, Mojica et al. 2003), the information on reproductive aspects of their populations along the coast of Brazil is still very limited (e.g. Menezes et al. 2004), and sufficient data is not available for the comparison of populations and species. The analysis of how reproductive parameters vary among species and among populations of the same species can allow us to understand adaptive responses in the natural history of species and any differences between bisexual and unisexual species within these parameters.

In this study we analyzed some reproductive aspects of 16 coastal populations belonging to the five aforementioned species of the genus Ameivula and Contomastix (Harvey et al. 2012) from different restinga habitats along approximately $3000 \mathrm{~km}$ of extension of the eastern coast of Brazil. The study's goals were (1) to evaluate to what extent the reproductive aspects (mean clutch size produced by females, minimum size at maturity for females and males) vary geographically and among species; (2) to determine the mean volume of eggs and the relative clutch mass (clutch mass/female mass) of females of cnemidophorines in each locality; (3) to evaluate the extent to which body size influences female clutch and egg size in each population/ species; (4) to determine if there are differences between bisexual and unisexual species in the relationship between body size and clutch size; and (5) to determine if there is sexual dimorphism in body size for lizards in the studied populations.

\section{MATERIALS AND METHODS}

STUDY AREAS

Fieldwork was carried out in 15 areas of restinga habitats from Genipabu, in the state of Rio Grande do Norte, northeastern Brazil, to Florianópolis, in the state of Santa Catarina, southern Brazil along approximately $3000 \mathrm{~km}$ of coast (Fig. 1, 2). 


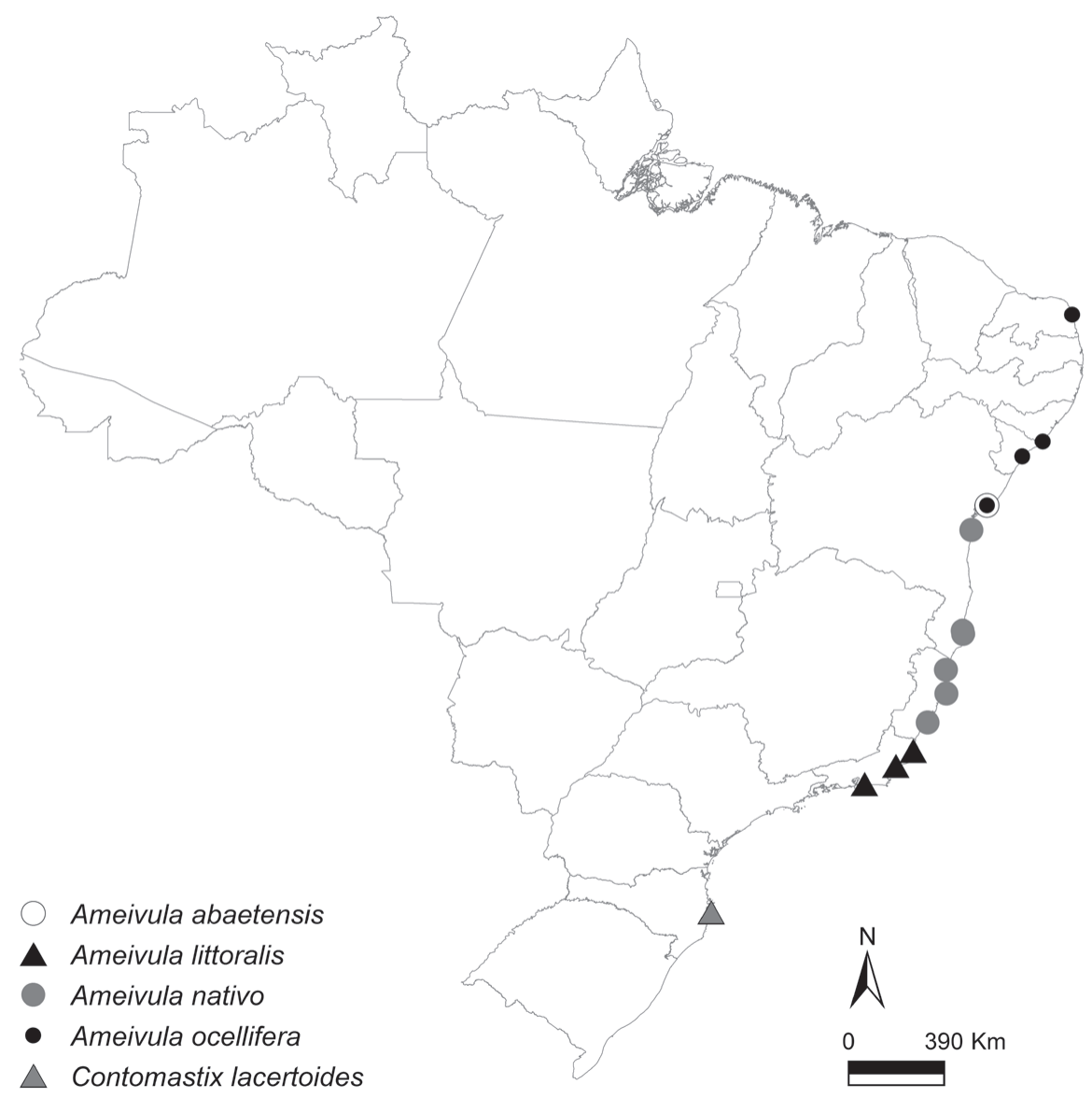

Figure 1 - Distribution of Ameivula and Contomastix species studied in the restingas along the eastern coast of Brazil.

\begin{tabular}{ccc}
\hline \hline Species & Locality & Coordinates \\
\hline \hline C. lacertoides & Joaquina, SC & $27^{\circ} 35^{\prime} \mathrm{S} ; 48^{\circ} 35^{\prime} \mathrm{W}$ \\
\hline \multirow{3}{*}{ A. littoralis } & Barra de Maricá, RJ & $22^{\circ} 57^{\prime} \mathrm{S} ; 42^{\circ} 50^{\prime} \mathrm{W}$ \\
& Jurubatiba, RJ & $22^{\circ} 17^{\prime} \mathrm{S} ; 41^{\circ} 41^{\prime} \mathrm{W}$ \\
& Grussaí, RJ & $21^{\circ} 44^{\prime} \mathrm{S} ; 41^{\circ} 02^{\prime} \mathrm{W}$ \\
\hline Setiba, ES & $20^{\circ} 34^{\prime} \mathrm{S} ; 40^{\circ} 27^{\prime} \mathrm{W}$ \\
A. nativo & Comboios, ES & $19^{\circ} 42^{\prime} \mathrm{S} ; 39^{\circ} 57^{\prime} \mathrm{W}$ \\
& Guriri, ES & $18^{\circ} 41^{\prime} \mathrm{S} ; 39^{\circ} 45^{\prime} \mathrm{W}$ \\
& Guaratiba, BA & $17^{\circ} 25^{\prime} \mathrm{S} ; 39^{\circ} 12^{\prime} \mathrm{W}$ \\
& Prado, BA & $17^{\circ} 18^{\prime} \mathrm{S} ; 39^{\circ} 13^{\prime} \mathrm{W}$ \\
\hline Abaetensis & $13^{\circ} 36^{\prime} \mathrm{S} ; 38^{\circ} 54^{\prime} \mathrm{W}$ \\
\hline Península de Maraú, BA & $12^{\circ} 38^{\prime} \mathrm{S} ; 38^{\circ} 04^{\prime} \mathrm{W}$ \\
\hline A. ocellifera & Guarajuba, BA & $12^{\circ} 38^{\prime} \mathrm{S} ; 38^{\circ} 04^{\prime} \mathrm{W}$ \\
& Guarajuba, BA & $10^{\circ} 56^{\prime} \mathrm{S} ; 37^{\circ} 01^{\prime} \mathrm{W}$ \\
& Praia do Porto, SE & $10^{\circ} 54^{\prime} \mathrm{S} ; 37^{\circ} 02^{\prime} \mathrm{W}$ \\
& Barra dos Coqueiros, SE & $10^{\circ} 21^{\prime} \mathrm{S} ; 36^{\circ} 18^{\prime} \mathrm{W}$ \\
\hline \hline
\end{tabular}

Figure 2 - Geographic distribution of the five cnemidophorine species living in restinga habitats along the eastern coast of Brazil. Brazilian State codes are: SC - Santa Catarina, RJ - Rio de Janeiro, ES - Espírito Santo, BA - Bahia, SE - Sergipe, AL - Alagoas, RN - Rio Grande do Norte. 
Restingas are sandy strips located between the sea and the mountains of the Brazilian eastern seaboard and are part of the Atlantic Forest biome. This type of habitat originated in the Quaternary, resulting from successive marine regressions which occurred throughout the Holocene and Pleistocene periods (Muehe 1983, Perrin 1984, Suguio and Tessler 1984).

\section{SAmPling Methods AND Data Analyses}

Collections were all done during the rainy season (October-May), within three years (2004-2006, depending on the area), in order to reduce the possible influence of weather and seasonality on reproductive patterns.

We followed the procedures of the Society for the Study of Amphibians and Reptiles outlined in the Guidelines for Use of Life of Amphibians and Reptiles in Field Research, which recommend the use of anesthetics prior to euthanizing the animals. Lizards were collected with rubber bands or pellet rifles, euthanized with ether and immediately fixed in $10 \%$ formalin. We measured the snout-vent length (SVL) of the lizards using a Vernier caliper (to the nearest $0.1 \mathrm{~mm}$ ) and weighed them using a Pesola spring balance (to the nearest $0.001 \mathrm{~g}$ ) or a dynamometer (to the nearest $0.5 \mathrm{~g}$ ).

In the laboratory, we dissected individuals for gonadal analysis. For each female, we recorded the number of vitellogenic follicles in each ovary, presence of corpora lutea, and number and size of oviductal eggs. Follicles were considered vittelogenic when they were yellow and $\geq 1.4 \mathrm{~mm}$ in diameter. We measured the length and width of each egg and estimated its volume using the formula for the ellipsoid: $\frac{4}{3} \pi\left(\frac{L}{2}\right)\left(\frac{W}{2}\right)^{2}$ (Dunham 1983). Females with vitellogenic follicles, oviductal eggs and/or corpora lutea were considered reproductive.

For males, the length and the width of each testicle were measured, and the volume of each testicle was estimated using the formula for the ellipsoid. To determine if males were sexually mature, the left testicle and epididymis of each male were dehydrated, subjected to histological sections of $5 \mu \mathrm{m}$ and stained with hematoxylineosin. The presence of spermatozoa in seminiferous tubules and/or epididymis was used to differentiate between adults and juveniles.

Mean clutch size was estimated based on the number of oviductal eggs or vitellogenic follicles. The simultaneous occurrence of vitellogenic follicles and oviductal eggs or of vitellogenic follicles and corpora lutea was considered as evidence of the production of at least two clutches per female per reproductive season (multiple clutches).

Relative clutch mass (RCM) was calculated as clutch mass divided by total mass (body mass + clutch mass). The effect of female body size (SVL) on clutch size for each population and on clutch size, egg volume and relative clutch mass (arcsine-transformed) for each species (pooling data from different populations for each species) was estimated using simple regression analysis or, when values were not normally distributed, Spearman Correlation (Zar 1999).

The differences in mean clutch size, egg volume and relative clutch mass among different populations of a species and among females of different species were tested by analysis of covariance (ANCOVA, using female body size as the covariate) followed by Scheffe tests for multiple comparisons (Zar 1999).

The differences in adult male and female SVL among populations/species were also evaluated using ANOVA and post-hoc Scheffe test to determine what pairs of populations/species differed in their coastal distribution (Zar 1999). An ANOVA and post-hoc Scheffe test were also used to compare minimum size at maturity (MSM) values among lizard populations/species. The relationships between female MSM and mean SVL and clutch size were tested for the species studied using simple regression analysis (Zar 1999). 
All data were tested for homocedasticity of variances and for normality of distributions before performing statistical analyses. Due to the wide variation in SVL and clutch size of some populations, these variables were log-transformed.

\section{RESULTS}

\section{INTRASPECIFIC COMPARISONS}

Among populations within each species, mean adult female SVL differed significantly for $A$. nativo (ANOVA, $\left.\mathrm{F}_{4,132}=5.298, \mathrm{r}^{2}=0.138, \mathrm{P}<0.05\right)$ and for A. ocellifera (ANOVA, $\mathrm{F}_{3,63}=3.469, \mathrm{r}^{2}=0.142$, $\mathrm{P}<0.05$ ). Among A. nativo populations, females from Setiba (ES) were significantly larger than those of Guriri (ES) (Post Hoc Scheffe, $\mathrm{P}<0.05$ ) and Guaratiba (BA) (Post Hoc Scheffe, $\mathrm{P}<0.05$ ) (Fig. 3). Because of the small sample of adult females $(n=3)$, the population of Prado was not included in this analysis. Among A. ocellifera populations, the Post Hoc Scheffe did not indicate significant differences in female mean SVL, although the probability value for females from Barra dos Coqueiros (SE) and Guarajuba (BA) was near significance $(\mathrm{P}=0.076)$. As a result of the small sample size of adult females $(\mathrm{n}=3)$, the population of Genipabu $(\mathrm{RN})$ was not included in this analysis.

The minimum size at maturity (MSM) was similar among populations of a given species (Fig. 3 and 4). The mean clutch size differed significantly among populations of $A$. littoralis (ANCOVA, $\mathrm{F}_{2,34}$ $=3.860, \mathrm{r}^{2}=0.187, \mathrm{P}<0.05$ ), with Grussaí (RJ) and Maricá (RJ) being the populations that differed from each other (Post Hoc Scheffe, $\mathrm{P}<0,05$ ), and for $C$. nativo (ANCOVA, $\mathrm{F}_{4,76}=2.752, \mathrm{r}^{2}=0.169, \mathrm{P}<0.05$ ) (Fig. 3). Post Hoc Scheffe did not indicate differences in CS among populations of $C$. nativo, although the difference in CS between the populations of Comboios (ES) and Maraú (BA) was close to significance (Post Hoc Scheffe, $\mathrm{P}=0.077$ ). The population of Prado (BA) was not included in this analysis due to the small sample size of adult females $(n=3)$.
Except for the populations of $A$. nativo from Guaratiba (Regression Analysis, $\mathrm{F}_{1,35}=6.528, \mathrm{r}^{2}=$ $0.157, \mathrm{P}<0.05)$ and of A. abaetensis from Guarajuba (Regression Analysis, $\mathrm{F}_{1,3}=13.414, \mathrm{r}^{2}=0.817, \mathrm{P}<$ $0.05)$, there was no relationship between clutch size and female SVL for any of the populations studied (all P values $>0.05$ ). When data of CS from different populations were pooled for each species, there was a positive and significant effect of female size on clutch size for A. nativo (Regression Analysis, $\mathrm{F}_{1,84}$ $\left.=4.487, \mathrm{r}^{2}=0.051, \mathrm{P}<0.05\right)$.

Female body size was positively and significantly correlated with egg volume for the populations of $A$. ocellifera (Regression Analysis, $\mathrm{F}_{1,12}=13.745$, $\mathrm{r}^{2}=0.534, \mathrm{P}<0.05$, pooled data) and with relative clutch mass for the populations of A. littoralis (Regression Analysis, $\mathrm{F}_{1,4}=12.707, \mathrm{r}^{2}=0.761$, $\mathrm{P}<0.05$, pooled data) and A. ocellifera (Spearman Correlation, $\mathrm{r}_{\mathrm{s}}=0.484, \mathrm{P}<0.05, \mathrm{n}=13$, pooled data).

Among populations within each species, mean adult male SVL differed significantly for $C$. littoralis (ANOVA, $\mathrm{F}_{2,46}=5.727, \mathrm{r}^{2}=0.199, \mathrm{P}<0.05$ ) and for C. ocellifer (ANOVA, $\mathrm{F}_{4,62}=3.327, \mathrm{r}^{2}=0.177$, $\mathrm{P}<0.05)$. Among $C$. littoralis populations, males from Maricá (RJ) were significantly larger than those from Grussaí (RJ) (Post Hoc Scheffe, $\mathrm{P}<0.05$ ) and Jurubatiba (RJ) (Post Hoc Scheffe, $\mathrm{P}<0.05$ ) (Fig. 6). Among C. ocellifer populations, the Post Hoc Scheffe did not indicate significant differences in male mean SVL.

\section{INTERSPECIFIC COMPARISONS}

The mean SVL of adult females varied from 52 to $63 \mathrm{~mm}$ and differed significantly among species (ANOVA, $\mathrm{F}_{4,272}=30.597, \mathrm{r}^{2}=0.533, \mathrm{P}<0.001$ ) (Fig. 3), with females of A. littoralis being significantly larger than $A$. nativo, and females of A. ocellifera smaller than the others (Post Hoc Scheffe, $\mathrm{P}<0.05$ ) (Fig. 3).

The minimum size at maturity (MSM) was similar among species (ANOVA: $\mathrm{F}_{4,9}=11.096$, $\left.\mathrm{r}^{2}=0.831, \mathrm{P}<0.05\right)$ (Fig. 3, 4), except for A. littoralis 


\begin{tabular}{|c|c|c|c|c|c|c|c|c|c|c|}
\hline Species & Locality & MSM & SVL & $\begin{array}{c}\text { Clutch } \\
\text { size }\end{array}$ & Egg length & Egg width & Egg volume & RCM & Frequency & $\begin{array}{l}\text { Multiple } \\
\text { clutches }\end{array}$ \\
\hline 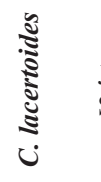 & $\begin{array}{l}\text { Joaquina, } \\
\text { SC }\end{array}$ & 51.1 & $\begin{array}{c}\mathbf{5 7 . 9} \pm \mathbf{4 . 2} \\
(51.1-65.8) \\
\mathrm{N}=14\end{array}$ & $\begin{array}{c}\mathbf{2 . 5} \pm \mathbf{1 . 1} \\
(1-4) \\
\mathrm{N}=13\end{array}$ & - & - & - & - & $\begin{array}{l}92.8 \% \\
(13 / 14)\end{array}$ & no \\
\hline \multirow{3}{*}{ 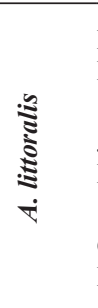 } & $\begin{array}{l}\text { Maricá, } \\
\text { RJ }\end{array}$ & 56.6 & $\begin{array}{c}\mathbf{6 2 . 6} \pm \mathbf{4 . 4} \\
(56.6-74.3) \\
\mathrm{N}=19\end{array}$ & $\begin{array}{c}\mathbf{1 . 9} \pm \mathbf{0 . 7} \\
(1-4) \\
\mathrm{N}=15\end{array}$ & $\begin{array}{c}\mathbf{1 8 . 8} \pm \mathbf{0 . 9} \\
(18.2-19.5) \\
\mathrm{N}=2\end{array}$ & $\begin{array}{c}\mathbf{8 . 5} \pm \mathbf{0 . 6} \\
(8.1-9.0) \\
\mathrm{N}=2\end{array}$ & $\begin{array}{c}\mathbf{7 2 5 . 7} \pm \mathbf{1 4 2 . 6} \\
(624.9-826.6) \\
\mathrm{N}=2\end{array}$ & $\begin{array}{l}\mathbf{0 . 1 9 0} \\
\mathrm{N}=1\end{array}$ & $\begin{array}{l}78.9 \% \\
(15 / 19)\end{array}$ & $\begin{array}{l}6.7 \% \\
(1 / 15)\end{array}$ \\
\hline & $\begin{array}{l}\text { Jurubatiba, } \\
\text { RJ }\end{array}$ & 57.7 & $\begin{array}{c}\mathbf{6 2 . 4} \pm \mathbf{3 . 5} \\
(55.2-69.3) \\
\mathrm{N}=19\end{array}$ & $\begin{array}{c}\mathbf{1 . 9} \pm \mathbf{1 . 2} \\
(1-5) \\
\mathrm{N}=15\end{array}$ & $\begin{array}{c}\mathbf{1 7 . 9} \pm \mathbf{1 . 9} \\
(15.0-20.0) \\
\mathrm{N}=5\end{array}$ & $\begin{array}{c}\mathbf{8 . 2} \pm \mathbf{0 . 7} \\
(7.0-8.8) \\
\mathrm{N}=5\end{array}$ & $\begin{array}{c}\mathbf{6 2 6 . 8} \pm \mathbf{9 6 . 2} \\
(482.1-721.0) \\
\mathrm{N}=5\end{array}$ & $\begin{array}{c}\mathbf{0 . 1 2 9} \pm \mathbf{0 . 0 2 7} \\
(0.084-0.151) \\
\mathrm{N}=5\end{array}$ & $\begin{array}{c}78.9 \% \\
(15 / 19)\end{array}$ & $\begin{array}{l}14.3 \% \\
(2 / 14)\end{array}$ \\
\hline & $\begin{array}{l}\text { Grussaí, } \\
\text { RJ }\end{array}$ & 54.1 & $\begin{array}{c}\mathbf{6 2 . 9} \pm \mathbf{6 . 1} \\
(54.1-69.2) \\
\mathrm{N}=10\end{array}$ & $\begin{array}{c}\mathbf{3 . 0} \pm \mathbf{1 . 0} \\
(1-4) \\
\mathrm{N}=9\end{array}$ & - & - & - & - & $\begin{array}{l}90.0 \% \\
(9 / 10)\end{array}$ & no \\
\hline \multirow{6}{*}{ 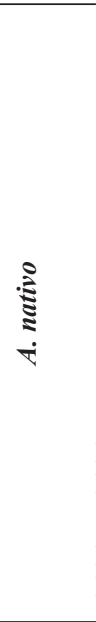 } & $\begin{array}{l}\text { Setiba, } \\
\text { ES }\end{array}$ & 50.6 & $\begin{array}{c}\mathbf{6 0 . 4} \pm \mathbf{8 . 6} \\
(47.2-79.5) \\
N=39\end{array}$ & $\begin{array}{c}\mathbf{2 . 3} \pm \mathbf{1 . 0} \\
(1-4) \\
\mathrm{N}=20\end{array}$ & $\begin{array}{c}\mathbf{1 8 . 0} \pm \mathbf{0 . 6} \\
(17.2-18.5) \\
\mathrm{N}=4\end{array}$ & $\begin{array}{c}\mathbf{8 . 7} \pm \mathbf{0 . 6} \\
(7.9-9.2) \\
\mathrm{N}=4\end{array}$ & $\begin{array}{c}\text { 711.6 } \pm \mathbf{1 1 3 . 4} \\
(594.4-819.4) \\
\mathrm{N}=4\end{array}$ & $\begin{array}{c}\mathbf{0 . 1 8 2} \pm \mathbf{0 . 0 0 2} \\
(0.181-0.184) \\
\mathrm{N}=2\end{array}$ & $\begin{array}{l}51.3 \% \\
(20 / 39)\end{array}$ & $\begin{array}{l}10.0 \% \\
(2 / 20)\end{array}$ \\
\hline & $\begin{array}{l}\text { Comboios, } \\
\text { ES }\end{array}$ & 51.1 & $\begin{array}{c}\mathbf{5 7 . 3} \pm \mathbf{5 . 8} \\
(47.2-63.7) \\
N=16\end{array}$ & $\begin{array}{c}\mathbf{1 . 6} \pm \mathbf{1 . 0} \\
(1-4) \\
\mathrm{N}=12\end{array}$ & $\begin{array}{c}\mathbf{1 5 . 5} \pm \mathbf{1 . 0} \\
(14.1-16.9) \\
\mathrm{N}=7\end{array}$ & $\begin{array}{c}\mathbf{8 . 0} \pm \mathbf{1 . 0} \\
(6.4-9.4) \\
\mathrm{N}=7\end{array}$ & $\begin{array}{c}\mathbf{5 2 6 . 4} \pm \mathbf{1 1 8 . 2} \\
(351.5-682.5) \\
\mathrm{N}=7\end{array}$ & $\begin{array}{c}\mathbf{0 . 1 2 6} \pm \mathbf{0 . 0 3 5} \\
(0.098-0.179) \\
\mathrm{N}=5\end{array}$ & $\begin{array}{c}75.0 \% \\
(12 / 16)\end{array}$ & $\begin{array}{l}58.3 \% \\
(7 / 12)\end{array}$ \\
\hline & Guriri, & 46.9 & $\begin{array}{c}\mathbf{5 4 . 1} \pm \mathbf{5 . 8} \\
(46.9-65.1) \\
\mathrm{N}=20\end{array}$ & $\begin{array}{c}\mathbf{1 . 3} \pm \mathbf{0 . 8} \\
(1-3) \\
\mathrm{N}=7\end{array}$ & - & - & - & - & $\begin{array}{l}35.0 \% \\
(7 / 20)\end{array}$ & no \\
\hline & $\begin{array}{l}\text { Guaratiba, } \\
\text { BA }\end{array}$ & 48.8 & $\begin{array}{c}\mathbf{5 5 . 2} \pm \mathbf{5 . 0} \\
(46.9-67.4) \\
\mathrm{N}=54\end{array}$ & $\begin{array}{c}\mathbf{2 . 2} \pm \mathbf{0 . 9} \\
(1-4) \\
\mathrm{N}=37\end{array}$ & $\begin{array}{c}\mathbf{1 7 . 1} \pm \mathbf{1 . 2} \\
(15.3-18.0) \\
\mathrm{N}=4\end{array}$ & $\begin{array}{c}\mathbf{7 . 7} \pm \mathbf{0 . 2} \\
(7.4-7.8) \\
\mathrm{N}=4\end{array}$ & $\begin{array}{c}\mathbf{5 3 3 . 1} \pm \mathbf{6 3 . 4} \\
(438.5-573.1) \\
\mathrm{N}=4\end{array}$ & $\begin{array}{c}\mathbf{0 . 2 0 5} \pm \mathbf{0 . 0 8 4} \\
(0.117-0.284) \\
\mathrm{N}=3\end{array}$ & $\begin{array}{c}68.5 \% \\
(37 / 54)\end{array}$ & $\begin{array}{l}2.7 \% \\
(1 / 37)\end{array}$ \\
\hline & $\begin{array}{l}\text { Prado, } \\
\text { BA }\end{array}$ & $* *$ & $\begin{array}{c}\mathbf{6 4 . 7} \pm \mathbf{1 . 0} \\
(63.6-65.4) \\
\mathrm{N}=3\end{array}$ & $\begin{array}{c}3.3 \pm 1.1 \\
(2-4) \\
N=3\end{array}$ & - & - & - & - & $\begin{array}{l}100 \% \\
(3 / 3)\end{array}$ & no \\
\hline & $\begin{array}{l}\text { Maraú, } \\
\text { BA }\end{array}$ & $* *$ & $\begin{array}{c}\mathbf{6 0 . 1} \pm \mathbf{1 . 7} \\
(57.7-62.2) \\
\mathrm{N}=8\end{array}$ & $\begin{array}{c}\mathbf{2 . 9} \pm \mathbf{1 . 2} \\
(1-4) \\
\mathrm{N}=8\end{array}$ & $\begin{array}{l}16.5 \\
N=1\end{array}$ & $\begin{array}{c}7.5 \\
N=1\end{array}$ & $\begin{array}{l}\mathbf{4 8 5 . 7} \\
N=1\end{array}$ & $\begin{array}{l}0.141 \\
N=1\end{array}$ & $\begin{array}{l}100 \% \\
(8 / 8)\end{array}$ & $\begin{array}{c}12.5 \% \\
(1 / 8)\end{array}$ \\
\hline 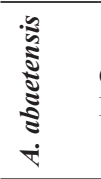 & $\begin{array}{l}\text { Guarajuba, } \\
\text { BA }\end{array}$ & 54.0 & $\begin{array}{c}\mathbf{6 1 . 6} \pm \mathbf{4 . 9} \\
(54.0-65.1) \\
\mathrm{N}=5\end{array}$ & $\begin{array}{c}\mathbf{4 . 0} \pm \mathbf{1 . 2} \\
(2-5) \\
\mathrm{N}=5\end{array}$ & - & - & - & - & $\begin{array}{l}100 \% \\
(5 / 5)\end{array}$ & no \\
\hline \multirow{5}{*}{ 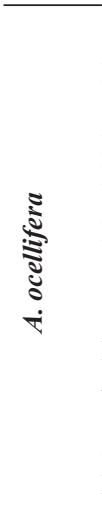 } & $\begin{array}{l}\text { Guarajuba, } \\
\text { BA }\end{array}$ & 45.9 & $\begin{array}{c}\mathbf{5 0 . 3} \pm \mathbf{2 . 8} \\
(45.9-56.4) \\
\mathrm{N}=18\end{array}$ & $\begin{array}{c}\mathbf{1 . 7} \pm \mathbf{0 . 9} \\
(1-3) \\
\mathrm{N}=19\end{array}$ & $\begin{array}{c}\mathbf{1 4 . 8} \pm \mathbf{2 . 0} \\
(12.1-16.8) \\
\mathrm{N}=4\end{array}$ & $\begin{array}{c}7.4 \pm \mathbf{0 . 9} \\
(6.8-8.8) \\
N=4\end{array}$ & $\begin{array}{c}\mathbf{4 3 6 . 1} \pm \mathbf{1 6 9 . 0} \\
(292.8-680.8) \\
\mathrm{N}=4\end{array}$ & $\begin{array}{c}\mathbf{0 . 1 2 9} \pm \mathbf{0 . 0 1 5} \\
(0.120-0.146) \\
\mathrm{N}=3\end{array}$ & $\begin{array}{c}100 \% \\
(19 / 19)\end{array}$ & $\begin{array}{l}10.5 \% \\
(2 / 19)\end{array}$ \\
\hline & $\begin{array}{l}\text { Praia do } \\
\text { Porto, SE }\end{array}$ & 48.2 & $\begin{array}{c}\mathbf{5 2 . 0} \pm \mathbf{2 . 4} \\
(48.2-58.1) \\
\mathrm{N}=26\end{array}$ & $\begin{array}{c}\mathbf{2 . 3} \pm \mathbf{1 . 1} \\
(1-4) \\
\mathrm{N}=24\end{array}$ & $\begin{array}{c}\mathbf{1 4 . 6} \pm \mathbf{1 . 6} \\
(13.2-17.7) \\
\mathrm{N}=6\end{array}$ & $\begin{array}{c}\mathbf{6 . 7} \pm \mathbf{0 . 6} \\
(5.7-7.4) \\
\mathrm{N}=6\end{array}$ & $\begin{array}{c}\mathbf{3 4 5 . 7} \pm \mathbf{\pm 5 7 . 0} \\
(232.9-392.6) \\
\mathrm{N}=6\end{array}$ & $\begin{array}{c}\mathbf{0 . 1 2 1} \pm \mathbf{0 . 0 1 6} \\
(0.105-0.151) \\
\mathrm{N}=6\end{array}$ & $\begin{array}{l}92.3 \% \\
(24 / 26)\end{array}$ & $\begin{array}{l}25.0 \% \\
(6 / 24)\end{array}$ \\
\hline & $\begin{array}{l}\text { Barra dos } \\
\text { Coqueiros, } \\
\text { SE }\end{array}$ & 50.5 & $\begin{array}{c}\mathbf{5 3 . 4} \pm \mathbf{2 . 6} \\
(50.5-57.7) \\
\mathrm{N}=6\end{array}$ & $\begin{array}{c}\mathbf{1 . 5} \pm \mathbf{0 . 5} \\
(1-2) \\
\mathrm{N}=6\end{array}$ & $\begin{array}{c}\mathbf{1 5 . 8} \pm \mathbf{1 . 0} \\
(15.1-16.5) \\
\mathrm{N}=2\end{array}$ & $\begin{array}{c}7.7 \pm 1.1 \\
(7.0-8.5) \\
N=2\end{array}$ & $\begin{array}{c}\mathbf{5 0 5 . 5} \pm \mathbf{1 6 7 . 3} \\
(387.2-623.9) \\
\mathrm{N}=2\end{array}$ & $\begin{array}{c}\mathbf{0 . 0 9 9} \pm \mathbf{0 . 0 0 1} \\
(0.098-0.100) \\
\mathrm{N}=2\end{array}$ & $\begin{array}{l}100 \% \\
(6 / 6)\end{array}$ & $\begin{array}{c}33.3 \% \\
(2 / 6)\end{array}$ \\
\hline & $\begin{array}{l}\text { Piaçabuçu, } \\
\text { AL }\end{array}$ & 48.1 & $\begin{array}{c}\mathbf{5 0 . 7} \pm \mathbf{2 . 1} \\
(46.2-53.4) \\
\mathrm{N}=17\end{array}$ & $\begin{array}{c}\mathbf{1 . 6} \pm \mathbf{0 . 8} \\
(1-4) \\
N=14\end{array}$ & $\begin{array}{c}\mathbf{1 4 . 2} \pm \mathbf{1 . 1} \\
(13.4-15.0) \\
\mathrm{N}=2\end{array}$ & $\begin{array}{c}7.2 \pm 0.6 \\
(6.8-7.6) \\
N=2\end{array}$ & $\begin{array}{c}\mathbf{3 8 4 . 0} \pm \mathbf{2 9 . 7} \\
(363.0-405.0) \\
\mathrm{N}=2\end{array}$ & $\begin{array}{c}\mathbf{0 . 1 0 8} \pm \mathbf{0 . 0 0 4} \\
(0.106-0.111) \\
\mathrm{N}=2\end{array}$ & $\begin{array}{c}82.3 \% \\
(14 / 17)\end{array}$ & $\begin{array}{l}7.1 \% \\
(1 / 14)\end{array}$ \\
\hline & $\begin{array}{l}\text { Genipabu, } \\
\text { RN }\end{array}$ & 47.9 & $\begin{array}{c}\mathbf{5 8 . 1} \pm \mathbf{8 . 9} \\
(47.9-64.3) \\
\mathrm{N}=3\end{array}$ & $\begin{array}{c}2.7 \pm 1.2 \\
(2-4) \\
N=3\end{array}$ & $\begin{array}{c}\mathbf{1 6 . 9} \pm \mathbf{1 . 6} \\
(15.8-18.1) \\
\mathrm{N}=2\end{array}$ & $\begin{array}{c}\mathbf{8 . 0} \pm \mathbf{0 . 1} \\
(7.9-8.1) \\
\mathrm{N}=2\end{array}$ & $\begin{array}{c}\mathbf{5 6 8 . 7} \pm \mathbf{7 4 . 5} \\
(516.0-621.5) \\
\mathrm{N}=2\end{array}$ & $\begin{array}{l}\mathbf{0 . 2 1 6} \\
\mathrm{N}=1\end{array}$ & $\begin{array}{l}100 \% \\
(3 / 3)\end{array}$ & $\begin{array}{c}33.3 \% \\
(1 / 3)\end{array}$ \\
\hline
\end{tabular}

Figure 3 - Minimum size at maturity (MSM, in mm), mean snout-vent length of adult females (SVL, in mm), mean clutch size, egg length (in $\mathrm{mm}$ ), egg width (in $\mathrm{mm}$ ), egg volume (in $\mathrm{mm}^{3}$ ), relative clutch mass (RCM), frequency of reproductive females (\%) and frequency of occurrence (\%) of multiple clutches for different populations and species of cnemidophorines along the eastern coast of Brazil. Values are represented by the arithmetic mean \pm one standard deviation with the range in parentheses and sample size below. State codes: SC - Santa Catarina, RJ - Rio de Janeiro, ES - Espírito Santo, BA - Bahia, SE - Sergipe, AL - Alagoas, RN - Rio Grande do Norte. 


\begin{tabular}{|c|c|c|c|c|c|}
\hline & C. lacertoides & A. littoralis & A. nativo & A. abaetensis & A. ocellifera \\
\hline \multicolumn{6}{|l|}{ Females } \\
\hline SVL & $\begin{array}{c}\mathbf{5 7 . 9} \pm \mathbf{4 . 2} \\
(51.1-65.8) \\
\mathrm{N}=14\end{array}$ & $\begin{array}{c}\mathbf{6 2 . 6} \pm \mathbf{4 . 4} \\
(54.1-74.3) \\
N=48\end{array}$ & $\begin{array}{c}\mathbf{5 7 . 2} \pm \mathbf{6 . 8} \\
(46.9-79.5) \\
N=140\end{array}$ & $\begin{array}{c}\mathbf{6 1 . 6} \pm \mathbf{5 . 0} \\
(54.0-65.1) \\
\mathrm{N}=5\end{array}$ & $\begin{array}{c}\mathbf{5 1 . 6} \pm \mathbf{3 . 2} \\
(45.9-64.3) \\
\mathrm{N}=70\end{array}$ \\
\hline MSM & 51.1 & 54.1 & 46.9 & 54.0 & 45.9 \\
\hline Clutch size & $\begin{array}{c}\mathbf{2 . 5} \pm \mathbf{1 . 1} \\
(1-4) \\
\mathrm{N}=13\end{array}$ & $\begin{array}{c}\mathbf{2 . 1} \pm \mathbf{1 . 1} \\
(1-5) \\
\mathrm{N}=39\end{array}$ & $\begin{array}{c}2.2 \pm \mathbf{1 . 1} \\
(1-4) \\
\mathrm{N}=87\end{array}$ & $\begin{array}{c}4.0 \pm 1.2 \\
(2-5) \\
N=5\end{array}$ & $\begin{array}{c}\mathbf{2 . 0} \pm \mathbf{1 . 0} \\
(1-4) \\
\mathrm{N}=66\end{array}$ \\
\hline RCM & - & $\begin{array}{c}\mathbf{0 . 1 3 9} \pm \mathbf{0 . 0 3 5} \\
(0.084-0.190) \\
N=6\end{array}$ & $\begin{array}{c}\mathbf{0 . 1 5 9} \pm \mathbf{0 . 0 5 7} \\
(0.098-0.284) \\
\mathrm{N}=11\end{array}$ & - & $\begin{array}{c}\mathbf{0 . 1 2 9} \pm \mathbf{0 . 0 2 7} \\
(0.098-0.216) \\
N=14\end{array}$ \\
\hline Egg volume & - & $\begin{array}{c}\mathbf{6 5 5 . 1} \pm \mathbf{1 0 9 . 1} \\
(482.1-826.6) \\
\mathrm{N}=7\end{array}$ & $\begin{array}{c}\mathbf{5 7 1 . 8} \pm \mathbf{1 2 6 . 6} \\
(351.5-819.4) \\
\mathrm{N}=16\end{array}$ & - & $\begin{array}{c}\mathbf{4 2 0 . 9} \pm \mathbf{1 2 4 . 4} \\
(232.9-680.8) \\
\mathrm{N}=16\end{array}$ \\
\hline Egg length & - & $\begin{array}{c}\mathbf{1 8 . 2} \pm \mathbf{1 . 6} \\
(15.0-20.0) \\
\mathrm{N}=7\end{array}$ & $\begin{array}{c}\mathbf{1 6 . 6} \pm \mathbf{1 . 4} \\
(14.1-18.5) \\
\mathrm{N}=16\end{array}$ & - & $\begin{array}{c}\mathbf{1 5 . 1} \pm \mathbf{1 . 7} \\
(12.1-18.1) \\
\mathrm{N}=16\end{array}$ \\
\hline Egg width & - & $\begin{array}{c}\mathbf{8 . 3} \pm \mathbf{0 . 7} \\
(7.0-9.0) \\
\mathrm{N}=7\end{array}$ & $\begin{array}{c}\mathbf{8 . 1} \pm \mathbf{0 . 8} \\
(6.4-9.4) \\
N=16\end{array}$ & - & $\begin{array}{c}7.2 \pm \mathbf{0 . 8} \\
(5.7-8.8) \\
N=16\end{array}$ \\
\hline CS X SVL & $\begin{array}{c}\mathrm{F}_{1.11}=1.185 \\
\mathrm{r}^{2}=0.097 \\
\mathrm{P}=0.300\end{array}$ & $\begin{array}{c}\mathrm{F}_{1.36}=0.265 \\
\mathrm{r}^{2}=0.007 \\
\mathrm{P}=0.610\end{array}$ & $\begin{array}{c}\mathrm{F}_{1.83}=5.521 \\
\mathrm{r}^{2}=0.062 \\
\mathbf{P}<\mathbf{0 . 0 5}\end{array}$ & $\begin{array}{c}\mathrm{F}_{1.3}=13.414 \\
\mathrm{r}^{2}=0.817 \\
\mathbf{P}<\mathbf{0 . 0 5}\end{array}$ & $\begin{array}{c}\mathrm{F}_{1.63}=1.739 \\
\mathrm{r}^{2}=0.027 \\
\mathrm{P}=0.192\end{array}$ \\
\hline RCM X SVL & - & $\begin{array}{c}\mathrm{F}_{1.4}=12.707 \\
\mathrm{r}^{2}=0.761 \\
\mathbf{P}<\mathbf{0 . 0 5}\end{array}$ & $\begin{array}{c}\mathrm{F}_{1.9}=0.027 \\
\mathrm{r}^{2}=0.003 \\
\mathrm{P}=0.873\end{array}$ & - & $\begin{array}{c}\mathrm{r}_{\mathrm{s}}=0.484^{*} \\
\mathbf{P}<\mathbf{0 . 0 5} \\
\mathrm{n}=13\end{array}$ \\
\hline egg volume X SVL & & $\begin{array}{c}\mathrm{F}_{1.4}=0.551 \\
\mathrm{r}^{2}=0.121 \\
\mathrm{P}=0.499\end{array}$ & $\begin{array}{c}\mathrm{F}_{1.10}=3.819 \\
\mathrm{r}^{2}=0.276 \\
\mathrm{P}=0.079\end{array}$ & - & $\begin{array}{c}\mathrm{F}_{1.12}=13.745 \\
\mathrm{r}^{2}=0.534 \\
\mathbf{P}<\mathbf{0 . 0 5}\end{array}$ \\
\hline $\begin{array}{l}\text { SVL among } \\
\text { populations ANOVA }\end{array}$ & - & $\begin{array}{c}\mathrm{F}_{2.45}=0.046 \\
\mathrm{r}^{2}=0.002 \\
\mathrm{P}=0.955\end{array}$ & $\begin{array}{c}\mathrm{F}_{4.132}=5.298 \\
\mathrm{r}^{2}=0.138 \\
\mathbf{P}<\mathbf{0 . 0 5}\end{array}$ & - & $\begin{array}{c}\mathrm{F}_{3.63}=3.469 \\
\mathrm{r}^{2}=0.142 \\
\mathbf{P}<\mathbf{0 . 0 5}\end{array}$ \\
\hline $\begin{array}{l}\text { CS among } \\
\text { populations } \\
\text { ANCOVA }\end{array}$ & - & $\begin{array}{c}\mathrm{F}_{2.34}=3.860 \\
\mathrm{r}^{2}=0.187 \\
\mathbf{P}<\mathbf{0 . 0 5}\end{array}$ & $\begin{array}{c}\mathrm{F}_{5.78}=2.724 \\
\mathrm{r}^{2}=0.197 \\
\mathbf{P}<\mathbf{0 . 0 5}\end{array}$ & - & $\begin{array}{c}\mathrm{F}_{4.59}=1.747 \\
\mathrm{r}^{2}=0.141 \\
\mathrm{P}=0.152\end{array}$ \\
\hline \multicolumn{6}{|l|}{ Males } \\
\hline SVL & $\begin{array}{c}\mathbf{5 5 . 4} \pm \mathbf{4 . 1} \\
(44.8-60.2) \\
\mathrm{N}=23\end{array}$ & $\begin{array}{c}\mathbf{6 5 . 6} \pm \mathbf{7 . 7} \\
(48.0-78.5) \\
N=49\end{array}$ & - & $\begin{array}{c}\mathbf{6 5 . 4} \pm \mathbf{5 . 2} \\
(57.8-73.3) \\
\mathrm{N}=9\end{array}$ & $\begin{array}{c}\mathbf{5 1 . 2} \pm \mathbf{5 . 9} \\
(41.3-67.9) \\
\mathrm{N}=67\end{array}$ \\
\hline Mean testis volume & $\begin{array}{c}\mathbf{2 2 . 9} \pm \mathbf{5 . 1} \\
(10.2-31.6) \\
\mathrm{N}=25\end{array}$ & $\begin{array}{c}\mathbf{2 6 . 8} \pm \mathbf{2 . 7} \\
(2.7-57.2) \\
N=45\end{array}$ & - & $\begin{array}{c}\mathbf{1 8 . 8} \pm \mathbf{9 . 9} \\
(3.0-33.4) \\
\mathrm{N}=8\end{array}$ & $\begin{array}{c}\mathbf{1 1 . 1} \pm \mathbf{4 . 3} \\
(3.1-23.3) \\
\mathrm{N}=53\end{array}$ \\
\hline $\begin{array}{l}\text { SVL between males } \\
\text { and females ANOVA }\end{array}$ & $\begin{array}{c}\mathrm{F}_{1.35}=3.237 \\
\mathrm{r}^{2}=0.085 \\
\mathrm{P}=0.081\end{array}$ & $\begin{array}{c}\mathrm{F}_{1.95}=5.389 \\
\mathrm{r}^{2}=0.054 \\
\mathbf{P}<\mathbf{0 . 0 5}\end{array}$ & - & $\begin{array}{c}\mathrm{F}_{1.12}=1.779 \\
\mathrm{r}^{2}=0.129 \\
\mathrm{P}=0.207\end{array}$ & $\begin{array}{c}\mathrm{F}_{1.135}=0.388 \\
\mathrm{r}^{2}=0.003 \\
\mathrm{P}=0.534\end{array}$ \\
\hline
\end{tabular}

* Spearman rank correlation

Figure 4 - Mean snout-vent length (SVL, in mm), minimum size at sexual maturity (MSM, in mm), mean clutch size, relative clutch mass ( $\mathrm{RCM}$ ), volume (in $\mathrm{mm}^{3}$ ), length and width (in $\mathrm{mm}$ ) of eggs, and testis volume (in $\mathrm{mm}^{3}$ ) for five cnemidophorines species studied along the eastern Brazilian coast. Values are represented by arithmetic mean \pm one standard deviation with the range in parentheses and sample size below. Simple regression analyses between clutch size (CS), RCM and egg volume and female SVL. Results of Analyses of Variance for differences in SVL between males and females. Analysis of Variance (ANOVA) of female SVL and Analysis of Covariance (ANCOVA) of CS among populations of each species. 


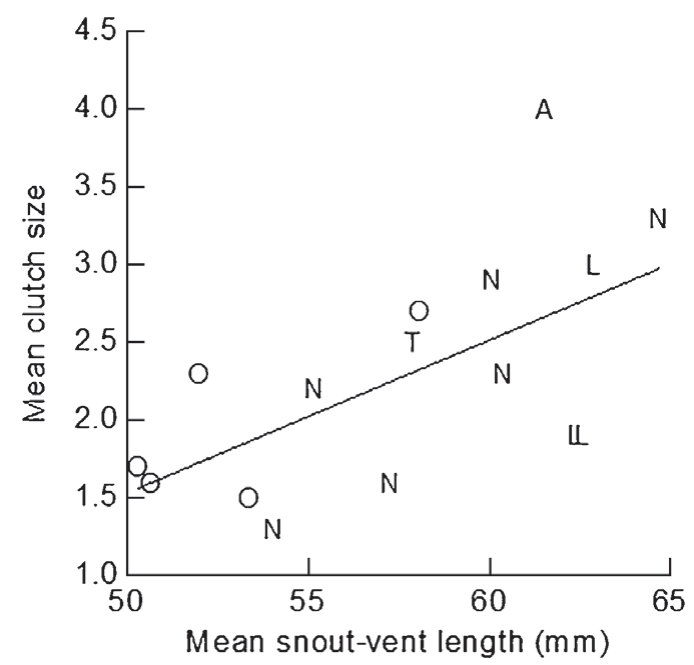

Figure 5 - Relationship between mean clutch size and mean snout-vent length $(\mathrm{mm})$ of different species and populations of cnemidophorines along the eastern coast of Brazil

that had a higher MSM when compared to A. nativo (Post Hoc Scheffe, $\mathrm{P}<0.05$ ) and to A. ocellifera (Post Hoc Scheffe, $\mathrm{P}<0.05)$. The minimum size at maturity was positively and significantly related to the mean body size among the species studied (Regression Analysis, $\left.\mathrm{F}_{1,3}=15.691, \mathrm{r}^{2}=0.839, \mathrm{P}<0.05\right)$.

The mean clutch size was approximately of 2 follicles/eggs for all species studied, except for A. abaetensis that had a mean clutch size of 4 follicles/eggs (Fig. 3, 4). The species studied differed significantly in clutch size (ANCOVA, $\left.\mathrm{F}_{4,200}=10.452, \mathrm{r}^{2}=0.106, \mathrm{P}<0.05\right)$, being the difference near significance only between $A$. abaetensis and $A$. littoralis (Post Hoc de Scheffe, $\mathrm{P}=0.056)$. Additionally, when mean clutch size of females of each population studied was regressed against mean SVL of the corresponding population, female size significantly explained the mean clutch size (Regression Analysis, $\mathrm{F}_{1,14}=$ 8.650, $\left.\mathrm{r}^{2}=0.382, \mathrm{P}<0.05\right)$ (Fig. 5).

There was no relationship between the minimum size at maturity and mean clutch size for the different populations/species of Ameivula studied (pooled data from all populations/species) (Regression Analysis, $\mathrm{F}_{1,12}=1.322, \mathrm{P}=0.273$ ).
Egg volume varied from 420 to $655 \mathrm{~mm}^{3}$ (Fig. 3, 4) and differed significantly among species (ANCOVA; $\mathrm{F}_{2,28}=13.038, \mathrm{r}^{2}=0.614, \mathrm{P}<0.05$ ), but the difference was near significance only between $A$. littoralis and A. ocellifera (Scheffe Post-Hoc test, $\mathrm{P}=0.096$ ). Relative clutch mass varied from 0.129 to 0.159 (Fig. 3,4) and did not differ significantly among species (ANCOVA, $\left.\mathrm{F}_{2,26}=1.601, \mathrm{r}^{2}=0.173, \mathrm{P}=0.217\right)$.

Mean SVL of adult males varied from 51 to $66 \mathrm{~mm}$ (Fig. 6) and differed significantly among species (ANOVA, $\mathrm{F}_{3,144}=54.365, \mathrm{r}^{2}=0.531, \mathrm{P}<$ 0.001 ), except for the pairs of species $A$. abaetensis - A. littoralis $(\mathrm{P}=1.000)$ and $A$. lacertoides - A. ocellifera $(\mathrm{P}=0.059)$ which did not differ.

There was no significant differences in mean SVL between adult males and females for the set of species studied, except for A. littoralis (pooled data from three populations, $F_{1,95}=5.389, \mathrm{r}^{2}=0.054$, $\mathrm{P}<0.05)$, in which males were larger than females.

\section{DISCUSSION}

The reproductive traits of a population/species such as reproductive period, clutch size, egg size, testes volume and minimum size at maturity can be affected by different environmental factors such as temperature (Cruz 1996, Ramirez-Bautista et al. 2000, Rezende-Pinto et al. 2009), photoperiod (Ramirez-Bautista and Vitt 1998, Rezende-Pinto et al. 2009), rainfall (Rodríguez-Ramírez and Lewis 1991, Ramirez-Bautista et al. 2000, Van Sluys et al. 2010) and food availability (Fitch 1982). Occasional intra and interspecific differences in some of those characteristics can also be partially attributed to the differences in body size of the females (Vitt and Congdon 1978, Van Sluys et al. 2010). In the present study, the data indicated some intrapopulational differences in mean clutch size for A. littoralis and for A. nativo, similar to those reported for populations of other cnemidophorines species from different geographical areas, such as C. lemniscatus (e.g. Léon and Cova 1973, 


\begin{tabular}{|c|c|c|c|c|}
\hline Species & Populations & Males MSM & Males SVL & Mean testis volume \\
\hline C. lacertoides & Joaquina, SC & 444.8 & $\begin{array}{c}\mathbf{5 5 . 4} \pm \mathbf{4 . 1} \\
(44.8-60.2) \\
\mathrm{N}=23\end{array}$ & $\begin{array}{c}\mathbf{2 2 . 9} \pm \mathbf{5 . 1} \\
(10.2-31.6) \\
\mathrm{N}=25\end{array}$ \\
\hline \multirow{3}{*}{ A. littoralis } & Maricá, RJ & $59.4^{*}$ & $\begin{array}{c}\mathbf{6 9 . 2} \pm \mathbf{5 . 9} \\
(59.4-78.5) \\
\mathrm{N}=22\end{array}$ & $\begin{array}{c}\mathbf{3 5 . 0} \pm \mathbf{8 . 8} \\
(23.1-57.2) \\
\mathrm{N}=19\end{array}$ \\
\hline & Jurubatiba, RJ & 48.0 & $\begin{array}{c}\mathbf{6 3 . 4} \pm \mathbf{8 . 0} \\
(48.0-76.1) \\
N=18\end{array}$ & $\begin{array}{c}\mathbf{2 0 . 2} \pm \mathbf{7 . 9} \\
(2.7-33.6) \\
N=18\end{array}$ \\
\hline & Grussaí, RJ & 54.1 & $\begin{array}{c}\mathbf{6 0 . 9} \pm \mathbf{7 . 8} \\
(48.6-71.6) \\
\mathrm{N}=9\end{array}$ & $\begin{array}{c}\mathbf{2 2 . 3} \pm \mathbf{1 1 . 4} \\
(8.5-40.1) \\
\mathrm{N}=8\end{array}$ \\
\hline A. abaetensis & Guarajuba, BA & $57.8^{*}$ & $\begin{array}{c}\mathbf{6 5 . 4} \pm \mathbf{5 . 2} \\
(57.8-73.3) \\
N=9\end{array}$ & $\begin{array}{c}\mathbf{1 8 . 8} \pm \mathbf{9 . 9} \\
(3.0-33.4) \\
\mathrm{N}=8\end{array}$ \\
\hline \multirow{5}{*}{ A. ocellifera } & Guarajuba, BA & 41.3 & $\begin{array}{c}\mathbf{4 8 . 7} \pm \mathbf{4 . 7} \\
(41.3-57.4) \\
\mathrm{N}=18\end{array}$ & $\begin{array}{c}7.4 \pm 2.3 \\
(3.1-11.6) \\
N=15\end{array}$ \\
\hline & Praia do Porto, SE & 42.4 & $\begin{array}{c}\mathbf{5 3 . 6} \pm \mathbf{5 . 7} \\
(42.4-67.9) \\
\mathrm{N}=18\end{array}$ & $\begin{array}{c}\mathbf{1 1 . 5} \pm \mathbf{4 . 4} \\
(3.8-23.3) \\
\mathrm{N}=16\end{array}$ \\
\hline & $\begin{array}{c}\text { Barra dos Coqueiros, } \\
\text { SE }\end{array}$ & 45.0 & $\begin{array}{c}\mathbf{4 8 . 7} \pm \mathbf{3 . 9} \\
(41.8-53.5) \\
N=8\end{array}$ & $\begin{array}{c}9.8 \pm 2.9 \\
(6.1-12.7) \\
N=6\end{array}$ \\
\hline & Piaçabuçu, AL & 47.2 & $\begin{array}{c}\mathbf{5 1 . 0} \pm \mathbf{5 . 5} \\
(41.9-60.9) \\
\mathrm{N}=19\end{array}$ & $\begin{array}{c}\mathbf{1 3 . 8} \pm \mathbf{3 . 2} \\
(8.9-19.0) \\
\mathrm{N}=13\end{array}$ \\
\hline & Genipabu, RN & $58.4^{*}$ & $\begin{array}{c}\mathbf{5 7 . 2} \pm \mathbf{1 0 . 3} \\
(42.3-65.5) \\
\mathrm{N}=4\end{array}$ & $\begin{array}{c}\mathbf{1 7 . 6} \pm \mathbf{2 . 5} \\
(14.7-19.3) \\
\mathrm{N}=3\end{array}$ \\
\hline
\end{tabular}

* Samples of senescente adult males

Figure 6 - Minimum size at maturity (MSM, in mm), mean snout-vent length (SVL, in $\mathrm{mm}$ ) and mean testis volume $\left(\mathrm{mm}^{3}\right)$ of males of different species and populations of Ameivula and Contomastix along the eastern coast of Brazil. Values are represented by arithmetic mean \pm one standard deviation with the range in parentheses and sample size below. State codes: SC - Santa Catarina, RJ - Rio de Janeiro, ES - Espírito Santo, BA - Bahia, SE - Sergipe, AL - Alagoas, RN - Rio Grande do Norte.

Magnusson 1987, Castro 1994, Mojica et al. 2003) and A. ocellifera (e.g. Vitt 1983, Cruz 1996, Mesquita and Colli 2003b). However, when data were grouped, the mean clutch size was considerably similar (approximately two eggs) for all species, with the exception of $A$. abaetensis in Guarajuba, BA ( $\bar{\chi}=4.0$ eggs). The relatively high value of mean clutch size for $A$. abaetensis found in the present study needs to be further studied, since the sample of reproductive females was relatively small $(\mathrm{n}=5)$ and Dias et al. (2002) in the restinga of Abaeté, in the state of Bahia, recorded for the same species a mean clutch size of 1.4 eggs.
The literature on the species of cnemidophorines in South America shows that the clutch size for species in the genus ranges from 1 to 5 follicles/ eggs, with most being around 2 eggs (Fig. 7). Thus, according to our study, the clutch size of the species of Ameivula and Contomastix in restinga habitats on the east coast of Brazil is similar to that found for most cnemidophorines species studied in South America such as C. cryptus in the Brazilian Amazon $(\bar{\chi}=1.9$, Vitt et al. 1997) and C. lemniscatus in Colombia $(\bar{\chi}=2.2$, Mojica et al. 2003) (Fig. 7).

The small clutch size in cnemidophorines has been associated with the energetic cost of 


\begin{tabular}{|c|c|c|c|c|c|c|c|}
\hline Species & Locality & Female SVL & MSM & CS & RCM & Egg volume & Source \\
\hline \multirow{2}{*}{$\begin{array}{c}\text { Ameivula abaetensis } \\
\text { Dias et al. } 2002\end{array}$} & $\begin{array}{c}\text { Guarajuba, } \\
\text { Bahia (Brazil) }\end{array}$ & $\begin{array}{c}\mathbf{6 1 . 6} \pm \mathbf{4 . 9} \\
(54.0-65.1) \\
\mathrm{N}=5\end{array}$ & 54.0 & $\begin{array}{c}\mathbf{4 . 0} \pm \mathbf{1 . 2} \\
(2-5) \\
N=5\end{array}$ & - & - & $\begin{array}{l}\text { Present } \\
\text { study }\end{array}$ \\
\hline & $\begin{array}{l}\text { Abaeté, Bahia } \\
\text { (Brazil) }\end{array}$ & $\begin{array}{c}\mathbf{6 3 . 4} \pm \mathbf{2 . 7} \\
(59.6-67.8)\end{array}$ & 59.6 & $\begin{array}{c}\mathbf{1 . 4} \pm \mathbf{0 . 5} \\
\mathrm{N}=11\end{array}$ & - & - & $\begin{array}{l}\text { Dias et al. } \\
2002\end{array}$ \\
\hline \multirow{3}{*}{$\begin{array}{l}\text { Ameivula littoralis } \\
\text { Rocha et al. } 2000\end{array}$} & $\begin{array}{l}\text { Maricá, Rio de } \\
\text { Janeiro } \\
\text { (Brazil) }\end{array}$ & $\begin{array}{c}\mathbf{6 2 . 6} \pm \mathbf{4 . 4} \\
(56.6-74.3) \\
N=19\end{array}$ & 56.6 & $\begin{array}{c}\mathbf{1 . 9} \pm \mathbf{0 . 7} \\
(1-4) \\
\mathrm{N}=15\end{array}$ & $\begin{array}{l}\mathbf{0 . 1 9 0} \\
\mathrm{N}=1\end{array}$ & $\begin{array}{c}\mathbf{7 2 5 . 7} \pm \mathbf{1 4 2 . 6} \\
(624.9-826.6) \\
\mathrm{N}=2\end{array}$ & $\begin{array}{l}\text { Present } \\
\text { study }\end{array}$ \\
\hline & $\begin{array}{l}\text { Jurubatiba, Rio } \\
\text { de Janeiro } \\
\text { (Brazil) }\end{array}$ & $\begin{array}{c}\mathbf{6 2 . 4} \pm \mathbf{3 . 5} \\
(55.2-69.3) \\
\mathrm{N}=19\end{array}$ & 57.7 & $\begin{array}{c}\mathbf{1 . 9} \pm \mathbf{1 . 2} \\
(1-5) \\
N=15\end{array}$ & $\begin{array}{c}\mathbf{0 . 1 2 9} \pm \mathbf{0 . 0 2 7} \\
(0.084-0.151) \\
\mathrm{N}=5\end{array}$ & $\begin{array}{c}\mathbf{6 2 6 . 8} \pm \mathbf{9 6 . 2} \\
(482.1-721.0) \\
\mathrm{N}=5\end{array}$ & $\begin{array}{l}\text { Present } \\
\text { study }\end{array}$ \\
\hline & $\begin{array}{l}\text { Grussaí, Rio de } \\
\text { Janeiro } \\
\text { (Brazil) }\end{array}$ & $\begin{array}{c}\mathbf{6 2 . 9} \pm \mathbf{6 . 1} \\
(54.1-69.2) \\
\mathrm{N}=10\end{array}$ & 54.1 & $\begin{array}{c}\mathbf{3 . 0} \pm \mathbf{1 . 0} \\
(1-4) \\
N=9\end{array}$ & - & - & $\begin{array}{l}\text { Present } \\
\text { study }\end{array}$ \\
\hline $\begin{array}{l}\text { Ameivula mumbuca } \\
\text { Colli et al. } 2003\end{array}$ & $\begin{array}{c}\text { Tocantins, } \\
\text { Goiás } \\
\text { (Brazil) }\end{array}$ & $\begin{array}{c}\mathbf{4 9 . 5} \pm \mathbf{7 . 4} \\
\mathrm{N}=223\end{array}$ & 56.0 & $\mathrm{~N}=30$ & - & $\begin{array}{c}491.9 \pm 67.9 \\
N=5\end{array}$ & $\begin{array}{l}\text { Colli et al. } \\
2003\end{array}$ \\
\hline \multirow{6}{*}{$\begin{array}{l}\text { Ameivula nativo } \\
\text { Rocha et al. } 1997^{* * *}\end{array}$} & $\begin{array}{c}\text { Setiba, } \\
\text { Espírito Santo } \\
\text { (Brazil) }\end{array}$ & $\begin{array}{c}\mathbf{6 0 . 4} \pm \mathbf{8 . 6} \\
(47.2-79.5) \\
\mathrm{N}=39\end{array}$ & 50.6 & $\begin{array}{c}\mathbf{2 . 3} \pm \mathbf{1 . 0} \\
(1-4) \\
N=20\end{array}$ & $\begin{array}{c}\mathbf{0 . 1 8 2} \pm \mathbf{0 . 0 0 2} \\
(0.181-0.184) \\
N=2\end{array}$ & $\begin{array}{c}\mathbf{7 1 1 . 6} \pm \mathbf{1 1 3 . 4} \\
(594.4-819.4) \\
N=4\end{array}$ & $\begin{array}{l}\text { Present } \\
\text { study }\end{array}$ \\
\hline & $\begin{array}{l}\text { Comboios, } \\
\text { Espírito Santo } \\
\text { (Brazil) }\end{array}$ & $\begin{array}{c}\mathbf{5 7 . 3} \pm \mathbf{5 . 8} \\
(47.2-63.7) \\
\mathrm{N}=16\end{array}$ & 51.1 & $\begin{array}{c}\mathbf{1 . 6} \pm \mathbf{1 . 0} \\
(1-4) \\
N=12\end{array}$ & $\begin{array}{c}\mathbf{0 . 1 2 6} \pm \mathbf{0 . 0 3 5} \\
(0.098-0.179) \\
\mathrm{N}=5\end{array}$ & $\begin{array}{c}\mathbf{5 2 6 . 4} \pm \mathbf{1 1 8 . 2} \\
(351.5-682.5) \\
\mathrm{N}=7\end{array}$ & $\begin{array}{l}\text { Present } \\
\text { study }\end{array}$ \\
\hline & $\begin{array}{c}\text { Guriri, } \\
\text { Espírito Santo } \\
\text { (Brazil) }\end{array}$ & $\begin{array}{c}\mathbf{5 4 . 1} \pm \mathbf{5 . 8} \\
(46.9-65.1) \\
\mathrm{N}=20\end{array}$ & 46.9 & $\begin{array}{c}\mathbf{1 . 3} \pm \mathbf{0 . 8} \\
(1-3) \\
N=7\end{array}$ & - & - & $\begin{array}{l}\text { Present } \\
\text { study }\end{array}$ \\
\hline & $\begin{array}{c}\text { Guaratiba, } \\
\text { Bahia (Brazil) }\end{array}$ & $\begin{array}{c}\mathbf{5 5 . 2} \pm \mathbf{5 . 0} \\
(46.9-67.4) \\
\mathrm{N}=54\end{array}$ & 48.8 & $\begin{array}{c}\mathbf{2 . 2} \pm \mathbf{1 . 0} \\
(1-4) \\
N=37\end{array}$ & $\begin{array}{c}\mathbf{0 . 2 0 5} \pm \mathbf{0 . 0 8 4} \\
(0.117-0.284) \\
\mathrm{N}=3\end{array}$ & $\begin{array}{c}\mathbf{5 3 3 . 1} \pm \mathbf{6 3 . 4} \\
(438.5-573.1) \\
N=4\end{array}$ & $\begin{array}{l}\text { Present } \\
\text { study }\end{array}$ \\
\hline & $\begin{array}{l}\text { Prado, Bahia } \\
\quad \text { (Brazil) }\end{array}$ & $\begin{array}{c}\mathbf{6 4 . 7} \pm \mathbf{1 . 0} \\
(63.6-65.4) \\
N=3\end{array}$ & - & $\begin{array}{c}3.3 \pm 1.1 \\
(2-4) \\
N=3\end{array}$ & - & - & $\begin{array}{l}\text { Present } \\
\text { study }\end{array}$ \\
\hline & $\begin{array}{l}\text { Maraú, Bahia } \\
\text { (Brazil) }\end{array}$ & $\begin{array}{c}\mathbf{6 0 . 1} \pm \mathbf{1 . 7} \\
(57.7-62.2) \\
\mathrm{N}=8\end{array}$ & - & $\begin{array}{c}\mathbf{2 . 9} \pm \mathbf{1 . 2} \\
(1-4) \\
N=8\end{array}$ & $\begin{array}{l}\mathbf{0 . 1 4 1} \\
\mathrm{N}=1\end{array}$ & $\begin{array}{l}485.7 \\
N=1\end{array}$ & $\begin{array}{l}\text { Present } \\
\text { study }\end{array}$ \\
\hline \multirow{5}{*}{$\begin{array}{l}\text { Ameivula ocellifera } \\
\quad \text { (Spix 1825) }\end{array}$} & $\begin{array}{c}\text { Guarajuba, } \\
\text { Bahia (Brazil) }\end{array}$ & $\begin{array}{c}\mathbf{5 0 . 3} \pm \mathbf{2 . 8} \\
(45.9-56.4) \\
\mathrm{N}=18\end{array}$ & 45.9 & $\begin{array}{c}\mathbf{1 . 7} \pm \mathbf{0 . 9} \\
(1-3) \\
\mathrm{N}=19\end{array}$ & $\begin{array}{c}\mathbf{0 . 1 2 9} \pm \mathbf{0 . 0 1 5} \\
(0.120-0.146) \\
\mathrm{N}=3\end{array}$ & $\begin{array}{c}\mathbf{4 3 6 . 1} \pm \mathbf{1 6 9 . 0} \\
(292.8-680.8) \\
\mathrm{N}=4\end{array}$ & $\begin{array}{l}\text { Present } \\
\text { study }\end{array}$ \\
\hline & $\begin{array}{l}\text { Praia do Porto, } \\
\text { Sergipe (Brazil) }\end{array}$ & $\begin{array}{c}\mathbf{5 2 . 0} \pm \mathbf{2 . 4} \\
(48.2-58.1) \\
\mathrm{N}=26\end{array}$ & 48.2 & $\begin{array}{c}\mathbf{2 . 3} \pm \mathbf{1 . 1} \\
(1-4) \\
N=24\end{array}$ & $\begin{array}{c}\mathbf{0 . 1 2 1} \pm \mathbf{0 . 0 1 6} \\
(0.105-0.151) \\
\mathrm{N}=6\end{array}$ & $\begin{array}{c}\mathbf{3 4 5 . 7} \pm \mathbf{5 7 . 0} \\
(232.9-392.6) \\
N=6\end{array}$ & $\begin{array}{l}\text { Present } \\
\text { study }\end{array}$ \\
\hline & $\begin{array}{c}\text { Barra dos } \\
\text { Coqueiros, } \\
\text { Sergipe (Brazil) }\end{array}$ & $\begin{array}{c}\mathbf{5 3 . 4} \pm \mathbf{2 . 6} \\
(50.5-57.7) \\
N=6\end{array}$ & 50.5 & $\begin{array}{c}\mathbf{1 . 5} \pm \mathbf{0 . 5} \\
(1-2) \\
N=6\end{array}$ & $\begin{array}{c}\mathbf{0 . 0 9 9} \pm \mathbf{0 . 0 0 1} \\
(0.098-0.100) \\
\mathrm{N}=2\end{array}$ & $\begin{array}{c}\mathbf{5 0 5 . 5} \pm \mathbf{1 6 7 . 3} \\
(387.2-623.9) \\
\mathrm{N}=2\end{array}$ & $\begin{array}{l}\text { Present } \\
\text { study }\end{array}$ \\
\hline & $\begin{array}{c}\text { Piaçabuçu, } \\
\text { Alagoas } \\
\text { (Brazil) }\end{array}$ & $\begin{array}{c}\mathbf{5 0 . 7} \pm \mathbf{2 . 1} \\
(46.2-53.4) \\
\mathrm{N}=17\end{array}$ & 48.1 & $\begin{array}{c}\mathbf{1 . 6} \pm \mathbf{0 . 8} \\
(1-4) \\
N=14\end{array}$ & $\begin{array}{c}\mathbf{0 . 1 0 8} \pm \mathbf{0 . 0 0 4} \\
(0.106-0.111) \\
\mathrm{N}=2\end{array}$ & $\begin{array}{c}\mathbf{3 8 4 . 0} \pm \mathbf{2 9 . 7} \\
(363.0-405.0) \\
\mathrm{N}=2\end{array}$ & $\begin{array}{l}\text { Present } \\
\text { study }\end{array}$ \\
\hline & $\begin{array}{l}\text { Genipabu, } \\
\text { Rio Grande do } \\
\text { Norte (Brazil) }\end{array}$ & $\begin{array}{c}\mathbf{5 8 . 1} \pm \mathbf{8 . 9} \\
(47.9-64.3) \\
\mathrm{N}=3\end{array}$ & 47.9 & $\begin{array}{c}2.7 \pm \mathbf{1 . 2} \\
(2-4) \\
N=3\end{array}$ & $\begin{array}{l}\mathbf{0 . 2 1 6} \\
\mathrm{N}=1\end{array}$ & $\begin{array}{c}\mathbf{5 6 8 . 7} \pm \mathbf{7 4 . 5} \\
(516.0-621.5) \\
\mathrm{N}=2\end{array}$ & $\begin{array}{l}\text { Present } \\
\text { study }\end{array}$ \\
\hline
\end{tabular}

Figure 7 - Snout-vent length (SVL in $\mathrm{mm}$ ) of females, minimum size at maturity (MSM, in mm), mean clutch size (CS), relative clutch mass $(\mathrm{RCM})$ and egg volume (in $\mathrm{mm}^{3}$ ) of different species and populations of cnemidophorines in South America and the Caribbean based on data from the literature. Values are represented by arithmetic mean \pm one standard deviation with the range in parentheses and sample size below. 


\begin{tabular}{|c|c|c|c|c|c|c|c|}
\hline Species & Locality & Female SVL & MSM & CS & RCM & Egg volume & Source \\
\hline \multirow{8}{*}{$\begin{array}{l}\text { Ameivula ocellifera } \\
\quad \text { (Spix 1825) }\end{array}$} & $\begin{array}{l}\text { Cerrado } \\
\text { (Brazil) }\end{array}$ & $\begin{array}{c}\mathbf{5 9 . 4}+\mathbf{5 . 2} \\
\mathrm{N}=131\end{array}$ & 51.0 & $\begin{array}{c}\mathbf{2 . 1} \pm \mathbf{0 . 7} * \\
(1-4) \\
\mathrm{N}=41\end{array}$ & - & $\begin{array}{c}\mathbf{4 8 7 . 4} \pm \mathbf{1 3 9 . 0} \\
\mathrm{N}=8\end{array}$ & $\begin{array}{l}\text { Mesquita } \\
\text { and Colli, } \\
2003 b\end{array}$ \\
\hline & $\begin{array}{l}\text { Cerrado } \\
\text { (Brazil) }\end{array}$ & $\begin{array}{c}\mathbf{5 7 . 4} \pm \mathbf{1 . 0} \\
(51.0-62.0) \\
\mathrm{N}=12\end{array}$ & 51.0 & $\begin{array}{c}\mathbf{2 . 3} \pm \mathbf{0 . 2} \\
(1-3) \\
\mathrm{N}=12\end{array}$ & - & - & Vitt 1991 \\
\hline & $\begin{array}{l}\text { Caatinga } \\
\text { (Brazil) }\end{array}$ & $(52.0-74.0)$ & 52.0 & $\begin{array}{c}\mathbf{1 . 8} \pm \mathbf{0 . 5} \\
(1-3) \\
\mathrm{N}=23\end{array}$ & - & - & $\begin{array}{l}\text { Mesquita } \\
\text { and Colli } \\
\text { 2003a }\end{array}$ \\
\hline & $\begin{array}{l}\text { Morro do } \\
\text { Chapéu, Bahia } \\
\text { (Brazil) }\end{array}$ & $\begin{array}{c}\mathbf{6 3 . 4} \pm \mathbf{4 . 4} \\
(52.5-69.9) \\
\mathrm{N}=13\end{array}$ & 52.5 & $\begin{array}{c}\mathbf{2 . 7}+\mathbf{1 . 6} \\
(1-6) \\
\mathrm{N}=11\end{array}$ & - & $\begin{array}{c}\mathbf{4 9 4 . 1} \pm \mathbf{4 0 . 4} \\
(458.5-538.1) \\
\mathrm{N}=3\end{array}$ & $\begin{array}{l}\text { Menezes } \\
\text { et al. } 2011\end{array}$ \\
\hline & $\begin{array}{c}\text { Northeastern } \\
\text { Brazil }\end{array}$ & - & - & $\begin{array}{c}2.7 \\
(1-5) \\
\mathrm{N}=58\end{array}$ & - & 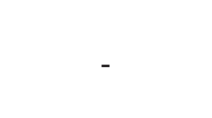 & Fitch 1985 \\
\hline & $\begin{array}{l}\text { Exu, } \\
\text { Pernambuco } \\
\text { (Brazil) }\end{array}$ & $\begin{array}{c}\mathbf{6 9 . 4} \pm \mathbf{0 . 5 * *} \\
(55.0 \text { a } 83.0) \\
\mathrm{N}=205\end{array}$ & 55.0 & $\begin{array}{c}2.7 \pm \mathbf{0 . 1} \\
(1-5) \\
\mathrm{N}=205\end{array}$ & $\begin{array}{c}\mathbf{0 . 2 0 7} \pm \mathbf{0 . 0 7} \\
\mathrm{N}=42\end{array}$ & - & Vitt 1983 \\
\hline & $\begin{array}{c}\text { Mataraca, } \\
\text { Paraíba (Brazil) }\end{array}$ & $\begin{array}{c}\mathbf{5 5 . 5} \pm \mathbf{1 . 1} * * \\
(42.4-67.0) \\
\mathrm{N}=29\end{array}$ & 42.4 & $\begin{array}{c}\mathbf{5 . 3} \pm \mathbf{0 . 4} \\
(1-10) \\
\mathrm{N}=154\end{array}$ & 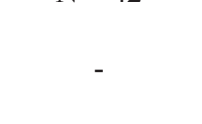 & - & $\begin{array}{l}\text { Santana et } \\
\text { al. } 2010\end{array}$ \\
\hline & $\begin{array}{c}\text { Salta } \\
\text { (Argentina) }\end{array}$ & $\begin{array}{c}\mathbf{5 7 . 7} \pm \mathbf{0 . 7} * * \\
(45.4-69.5) \\
\mathrm{N}=58\end{array}$ & 45.4 & $\begin{array}{c}\mathbf{2 . 4} \pm \mathbf{0 . 5} \\
(2-4) \\
\mathrm{N}=24\end{array}$ & $\begin{array}{c}\mathbf{0 . 2 1 4} \pm \mathbf{0 . 1 6 0} \\
\mathrm{N}=3\end{array}$ & - & Cruz 1996 \\
\hline $\begin{array}{c}\text { Ameivula parecis Colli } \\
\text { et al. } 2003\end{array}$ & $\begin{array}{l}\text { Cerrado } \\
\text { (Brazil) }\end{array}$ & $(72.0-89.0)$ & 72.0 & $\begin{array}{c}\mathbf{1 . 6} \pm \mathbf{0 . 5} \\
(1-2) \\
\mathrm{N}=11\end{array}$ & - & - & $\begin{array}{c}\text { Mesquita } \\
\text { and Colli } \\
\text { 2003a }\end{array}$ \\
\hline $\begin{array}{c}\text { Cnemidophorus } \\
\text { arenivagus Markezich } \\
\text { et al. } 1997\end{array}$ & $\begin{array}{c}\text { Paraguana } \\
\text { (Venezuela) }\end{array}$ & - & 58.0 & $\begin{array}{c}\mathbf{1 . 8} \\
(1-2) \\
\mathrm{N}=5 \\
\end{array}$ & - & - & $\begin{array}{l}\text { Markezich } \\
\text { et al. } 1997\end{array}$ \\
\hline $\begin{array}{c}\text { Cnemidophorus } \\
\text { arubensis Van Lidth de } \\
\text { Jeude } 1887\end{array}$ & $\begin{array}{c}\text { Aruba Island } \\
\text { (Netherlands } \\
\text { Antilles) }\end{array}$ & $\begin{array}{c}\sim 78.0 \\
(77.3-78.7) \\
N=146\end{array}$ & & $\begin{array}{c}\mathbf{1 . 0} \\
(1-2) \\
\mathrm{N}=38\end{array}$ & - & - & $\begin{array}{c}\text { Schall } \\
1983\end{array}$ \\
\hline \multirow{2}{*}{$\begin{array}{l}\text { Cnemidophorus } \\
\text { cryptus Cole and } \\
\text { Dessauer 1993*** }\end{array}$} & $\begin{array}{l}\text { Rio Xingu, } \\
\text { Amazonia } \\
\text { (Brazil) }\end{array}$ & $\begin{array}{c}\mathbf{6 4 . 9} \pm \mathbf{0 . 8} * * \\
(58.0-72.0) \\
\mathrm{N}=15\end{array}$ & 58.0 & $\begin{array}{c}\mathbf{1 . 9} \pm \mathbf{0 . 1} \\
(1-2) \\
\mathrm{N}=15\end{array}$ & - & - & $\begin{array}{l}\text { Vitt et al. } \\
1997\end{array}$ \\
\hline & $\begin{array}{l}\text { Amazon } \\
\text { Savanna } \\
\text { (Brazil) }\end{array}$ & $(50.0-65.0)$ & 50.0 & $\begin{array}{c}\mathbf{1 . 6} \pm \mathbf{0 . 6} \\
(1-3) \\
\mathrm{N}=16\end{array}$ & & & $\begin{array}{l}\text { Mesquita } \\
\text { and Colli } \\
\text { 2003a }\end{array}$ \\
\hline $\begin{array}{c}\text { Cnemidophorus } \\
\text { gramivagus McCrystal } \\
\text { and Dixon } 1987\end{array}$ & $\begin{array}{l}\text { Amazon } \\
\text { Savanna } \\
\text { (Brazil) }\end{array}$ & $(56.0-76)$ & 56.0 & $\begin{array}{c}\mathbf{1 . 7} \pm \mathbf{1 . 1} \\
(1-3) \\
\mathrm{N}=3\end{array}$ & - & - & $\begin{array}{l}\text { Mesquita } \\
\text { and Colli } \\
\text { 2003a }\end{array}$ \\
\hline \multirow{3}{*}{$\begin{array}{c}\text { Cnemidophorus } \\
\text { lemniscatus } \\
\text { lemniscatus (Linnaeus } \\
1758 \text { ) }\end{array}$} & $\begin{array}{c}\text { Lavrado, } \\
\text { Roraima } \\
\text { (Brazil) }\end{array}$ & $\begin{array}{c}54.7 \pm 0.9 * * \\
(46-61) \\
N=21\end{array}$ & 46.0 & $\begin{array}{c}\mathbf{1 . 7} \pm \mathbf{0 . 1} * \\
(1-4) \\
\mathrm{N}=32\end{array}$ & - & - & $\begin{array}{l}\text { Vitt et al. } \\
1997\end{array}$ \\
\hline & $\begin{array}{l}\text { Alter do Chão, } \\
\text { Pará } \\
\text { (Brazil) }\end{array}$ & $\begin{array}{c}\mathbf{5 7 . 0} \pm \mathbf{0 . 8} * * \\
(53-61) \\
\mathrm{N}=15\end{array}$ & 53.0 & $\begin{array}{c}\mathbf{1 . 7} \pm \mathbf{0 . 1} \\
(1-4) \\
\mathrm{N}=32\end{array}$ & - & - & $\begin{array}{l}\text { Vitt et al. } \\
1997\end{array}$ \\
\hline & $\begin{array}{c}\text { Curuá-Una, } \\
\text { Amazonia } \\
\text { (Brazil) }\end{array}$ & $\begin{array}{c}\mathbf{5 8 . 7} \pm \mathbf{3 . 2} * * \\
(55-65) \\
\mathrm{N}=3\end{array}$ & 55.0 & $\begin{array}{c}\mathbf{1 . 7} \pm \mathbf{0 . 1} * \\
(1-4) \\
\mathrm{N}=32\end{array}$ & - & - & $\begin{array}{l}\text { Vitt et al. } \\
1997\end{array}$ \\
\hline
\end{tabular}

Figure 7 - (continuation) 


\begin{tabular}{|c|c|c|c|c|c|c|c|}
\hline Species & Locality & Female SVL & MSM & CS & RCM & Egg volume & Source \\
\hline \multirow{6}{*}{$\begin{array}{c}\text { Cnemidophorus } \\
\text { lemniscatus } \\
\text { lemniscatus } \\
\text { (Linnaeus 1758) }\end{array}$} & $\begin{array}{l}\text { Caatinga } \\
\text { (Brazil) }\end{array}$ & $(49.0-61.0)$ & 49.0 & $\begin{array}{c}\mathbf{1 . 5} \pm \mathbf{0 . 5} \\
(1-2) \\
\mathrm{N}=16\end{array}$ & - & - & $\begin{array}{l}\text { Mesquita } \\
\text { and Colli } \\
2003 a\end{array}$ \\
\hline & $\begin{array}{c}\text { Cumaná } \\
\text { (Venezuela) }\end{array}$ & $\begin{array}{c}\mathbf{6 0 . 9} \\
(50.0-78.0) \\
N=194\end{array}$ & 50.0 & $\begin{array}{c}2.4 \\
(2-5) \\
N=56\end{array}$ & - & - & $\begin{array}{l}\text { Léon and } \\
\text { Cova } 1973\end{array}$ \\
\hline & $\begin{array}{c}\text { Cumaná } \\
\text { (Venezuela) }\end{array}$ & - & - & $\begin{array}{c}2.6 \\
(2-5) \\
N=94\end{array}$ & - & - & Fitch 1985 \\
\hline & $\begin{array}{c}\text { Carapito } \\
\text { (Venezuela) }\end{array}$ & - & - & $\begin{array}{c}\mathbf{2 . 0} \\
\mathrm{N}=5\end{array}$ & - & - & Fitch 1985 \\
\hline & $\begin{array}{l}\text { Kartabo } \\
\text { (Guyana) }\end{array}$ & - & - & $\begin{array}{c}\mathbf{2 . 0} \\
\mathrm{N}=5\end{array}$ & - & - & Fitch 1985 \\
\hline & $\begin{array}{l}\text { Piedecuesta, } \\
\text { Santander } \\
\text { (Colombia) }\end{array}$ & $\begin{array}{c}\mathbf{5 9 . 9} \pm \mathbf{3 . 9} \\
(55.0-65.0) \\
\mathrm{N}=117\end{array}$ & 50.0 & $\begin{array}{c}2.2 \pm 0.5 \\
(1-3) \\
N=46\end{array}$ & - & 345.6 & $\begin{array}{l}\text { Mojica et } \\
\text { al. } 2003\end{array}$ \\
\hline $\begin{array}{c}\text { Cnemidophorus } \\
\text { lemniscatus } \\
\text { splendidus Markezich, } \\
\text { Cole and Dessauer } \\
1997 \\
\end{array}$ & $\begin{array}{l}\text { Paraguana } \\
\text { (Venezuela) }\end{array}$ & $\begin{array}{c}\mathbf{6 1 . 2} \pm \mathbf{0 . 9} * * \\
(58.0-65.0) \\
\mathrm{N}=9\end{array}$ & 62.0 & $\begin{array}{c}2.3 \\
\mathbf{N}=\mathbf{3}\end{array}$ & - & - & $\begin{array}{l}\text { Markezich } \\
\text { et al. } 1997\end{array}$ \\
\hline $\begin{array}{l}\text { Cnemidophorus } \\
\text { murinus murinus } \\
\text { (Laurenti 1768) }\end{array}$ & $\begin{array}{c}\text { Bonaire Island, } \\
\text { (Netherlands } \\
\text { Antilles) }\end{array}$ & $\begin{array}{c}\mathbf{9 7 . 6}+\mathbf{6 . 7} \\
(85.0-116.0) \\
\mathrm{N}=513 \\
\end{array}$ & 85.0 & $\begin{array}{c}\mathbf{1 . 0} \\
(1-2) \\
\mathrm{N}=12\end{array}$ & $\begin{array}{c}\mathbf{0 . 1 2 6}+\mathbf{0 . 0 3 3} \\
\mathrm{N}=12\end{array}$ & - & $\begin{array}{c}\text { Dearing } \\
\text { and Schall } \\
1994 \\
\end{array}$ \\
\hline \multirow{2}{*}{$\begin{array}{c}\text { Contomastix } \\
\text { lacertoides Duméril } \\
\text { and Bibron } 1839\end{array}$} & $\begin{array}{l}\text { Joaquina, Santa } \\
\text { Catarina } \\
\text { (Brazil) }\end{array}$ & $\begin{array}{c}\mathbf{5 7 . 9} \pm \mathbf{4 . 2} \\
(51.1-65.8) \\
\mathrm{N}=14\end{array}$ & 51.1 & $\begin{array}{c}\mathbf{2 . 5} \pm \mathbf{1 . 1} \\
(1-4) \\
\mathrm{N}=13\end{array}$ & - & - & $\begin{array}{l}\text { Present } \\
\text { study }\end{array}$ \\
\hline & $\begin{array}{c}\text { Córdoba } \\
\text { (Argentina) }\end{array}$ & $\begin{array}{c}\mathbf{5 6 . 0} \\
(43.0-62.0) \\
\mathrm{N}=34 \\
\end{array}$ & 43.0 & $\begin{array}{c}3.0 \\
(1-4)\end{array}$ & - & - & $\begin{array}{c}\text { Aún and } \\
\text { Martori } \\
1996 \\
\end{array}$ \\
\hline $\begin{array}{l}\text { Contomastix serrana } \\
\text { Cei and Martori } 1991\end{array}$ & $\begin{array}{l}\text { Córdoba, } \\
\text { (Argentina) }\end{array}$ & $\begin{array}{c}\mathbf{5 6 . 0} \\
(42.0-60.0) \\
\mathrm{N}=57 \\
\end{array}$ & 42.0 & $\begin{array}{c}\mathbf{3 . 0} \\
(1-3)\end{array}$ & - & - & $\begin{array}{c}\text { Aún and } \\
\text { Martori } \\
1996 \\
\end{array}$ \\
\hline $\begin{array}{c}\text { Contomastix } \\
\text { vacariensis Feltrim } \\
\text { and Lema } 2000 \\
\end{array}$ & $\begin{array}{c}\text { Vacaria, } \\
\text { Rio Grande do } \\
\text { Sul (Brazil) } \\
\end{array}$ & $\begin{array}{c}\mathbf{7 0 . 0}+\mathbf{5 . 9} \\
(57.4-81.8) \\
\mathrm{N}=73 \\
\end{array}$ & 57.4 & $\begin{array}{c}\mathbf{4 . 1} \pm \mathbf{0 . 9} \\
(2-6) \\
\mathrm{N}=21 \\
\end{array}$ & $\begin{array}{c}\mathbf{0 . 1 8 3} \\
(0.16-0.22) \\
\mathrm{N}=3 \\
\end{array}$ & $\begin{array}{c}(227.0-638.9) \\
\mathrm{N}=22 \\
\end{array}$ & $\begin{array}{c}\text { Rezende- } \\
\text { Pinto et al. } \\
2009 \\
\end{array}$ \\
\hline
\end{tabular}

* Data were pooled (populations with * were grouped by the authors to obtain a single value of mean clutch size for the species studied).

** Data represented by mean + standard error

*** Unisexual species

\section{Figure 7 - (continuation)}

carrying the eggs, which probably selects for a smaller clutch mass, especially in active foraging species when compared with sedentary foragers (Vitt and Breitenbach 1993). In the present study, the volume of the eggs cannot be compared among populations of Ameivula due to the small number of ovigerous females in the populations' samples, but among the species studied, there was no difference in the volume of the eggs after removing the effect of female SVL, except for A. littoralis and A. ocellifera, which differed from each other. Clutch size and egg volume are parameters that are associated with the size of offspring produced and therefore with a presumed greater chance of hatchling and or juvenile survival. Concomitantly, the relative clutch mass influences the foraging behavior and the probability of a pregnant female of escaping from a predator, being associated with the female's ability to carry the clutch mass (Vitt and Price 1982). According to Vitt and Price (1982), 
RCM tends to be low in active foraging lizards and varies little among individuals or species.

There was no difference in the relative clutch mass among species studied. When compared with other species of cnemidophorines in South America, the values of RCM in this study are within the expected pattern (Fig. 7). When compared with sedentary foraging lizards $(\bar{\chi}=0.228 \pm 0.009, \mathrm{n}=$ 86, Vitt and Price 1982), the RCM of species studied was comparatively low $(\bar{\chi}=0.140 \pm 0.044, \mathrm{n}=31$, all species together). RCM represents a balance between the advantages of a high investment in reproduction and the costs associated with it in relation to foraging and escape behavior (Vitt and Price 1982), as has been demonstrated for some lizard species whose running speed was impaired by an increase in clutch mass (Shine 1980).

The minimum size at maturity for females was similar among populations of a given specie of coastal Ameivula and Contomastix (A. littoralis: 54.1 to $57.7 \mathrm{~mm}$, A. nativo: 46.9 to $51.1 \mathrm{~mm}, A$. ocellifera: 45.9 to $50.5 \mathrm{~mm}$ ). Also, among the studied species, female sexual maturity is reached at similar sizes (C. lacertoides: $51.1 \mathrm{~mm}$, A. littoralis: 54.1 mm, A. nativo: $46.9 \mathrm{~mm}$, A. abaetensis: $54.0 \mathrm{~mm}$, A. ocellifera: $45.9 \mathrm{~mm}$ ). Although the relationship between the minimum size at maturity and clutch size was not significant, there is a perceptible biological tendency in the species/populations studied for those who had larger minimum size at maturity to have slightly larger clutches.

For most species of lizards there is a general trend to increase clutch/litter size with the increase in female body size, both intra and interspecifically (Dunham et al. 1988, King 2000, Kiefer et al. 2008, Vrcibradic and Rocha 2011), and Ameivula and Contomastix spp. are no exception (e.g. Vitt 1983, Vitt et al. 1997, Mesquita and Colli 2003b, Mojica et al. 2003). Body size affected clutch size when females of different species were analyzed together, suggesting that larger females in general tend to produce larger clutches. Although the size of the female had a significant effect on clutch size produced, this effect was relatively small $(38 \%)$ as a result of the little variation in clutch size both within and between species (1 to 5 eggs). Among all the studied areas, only for the populations of A. nativo in Guaratiba and of A. abaetensis in Guarajuba (both in the state of Bahia) were found a significant effect of the body size on the clutch size of females. When the data for all populations were analyzed together, the effect of the female SVL on the clutch size remained significant for A. nativo, albeit relatively weak $(6 \%)$.

Sexual dimorphism has been reported in different populations and species of lizards; in some species, larger body size may be favored in females, due to its influence on clutch size and egg volume (e.g. Fitch 1981, Anderson and Vitt 1990, Rocha 2008). However, in other species, females are smaller than males, suggesting that they employ a greater investment of energy in the production of a clutch, thus having a smaller amount of energy available for growth (e.g. Rocha 1992, Van Sluys 1998). Also, in some cases females reach smaller sizes than males as a result of intrasexual selection, thus males obtain advantages with larger body size during agonistic interactions with other males (Fitch 1981, Vitt and Cooper 1985, Rocha 1992). In the present study, in general, we did not observe a clear tendency for sexual dimorphism in SVL for the species studied, with the exception of $A$. littoralis, where males were significantly larger than females.

We concluded that the five species of cnemidophorines studied (four bisexual and one parthenogenetic) had similar reproductive characteristics. Species presented small clutch size and low relative clutch mass; three of them had multiple clutches per year, similar to other species/ populations of the genus studied previously (e.g. Vitt 1983, Dearing and Schall 1994, Cruz 1996) and to other unisexual and bisexual species of the closely related genus Aspidoscelis (sensu Reeder et al. 2002) (e.g. Schall 1978, Ramírez-Bautista et al. 2000). 
Long-term studies of populations of these species are needed in order to gain knowledge about the survival of adults, the frequency of clutches per year, the time required for development from birth to sexual maturity, and environmental factors that may cause annual changes in mean clutch size of a given population/species as well as other important information about their reproductive biology.

\section{ACKNOWLEDGMENTS}

This study was supported by grants from the Fundação O Boticario de Proteção a Natureza to the authors and funds of the Conselho Nacional do Desenvolvimento Científico e Tecnológico (CNPq, Processes 304791/2010-5, 470265/2010-8 and 472287/2012-5) and of Fundação Carlos Chagas Filho de Amparo à Pesquisa do Estado do Rio de Janeiro (FAPERJ, Process E-26/102.765.2012) through the "Programa Cientistas do Nosso Estado" to CFDR. During this study VAM received a Graduate fellowship from CNPq. VAM also received a Post-Doctoral grant from FAPERJ (100.005/2009) and is currently associated with the Programa de PósGraduação em Ecologia of Universidade do Estado do Rio de Janeiro. We thank D. Vrcibradic who kindly revised the manuscript, offering helpful suggestions.

\section{RESUMO}

Analisamos alguns aspectos reprodutivos de 16 populações costeiras, pertencentes a cinco espécies de lagarto (A. ocellifera, A. abaetensis, A. nativo, A. littoralis e A. lacertoides) de diferentes habitats de restinga ao longo da costa leste da Brasil. Este estudo teve como objetivo avaliar em que extensão os aspectos reprodutivos variam geograficamente e entre as espécies. Para cada fêmea, registramos número de folículos vitelogênicos, tamanho e cor do maior folículo, presença e tamanho do corpo lúteo, e número e tamanho dos ovos no oviduto. O tamanho da ninhada de quase todas as populações/espécies costeiras de Ameivula teve pouca variação e a maioria das ninhadas era composta de dois ovos. Houve uma relação significativa entre o tamanho da fềmea e o tamanho médio da ninhada quando as fêmeas de diferentes espécies foram agrupadas. O volume médio dos ovos, entre as espécies, variou 420 a $655 \mathrm{~mm} 3$. A massa relativa da ninhada variou de 0,129 a 0,159 e não diferiu significativamente entre as espécies. Concluímos que as cinco espécies estudadas (quatro bissexuais e uma partenogenética) têm características reprodutivas semelhantes. A maioria apresentou ninhadas múltiplas, baixo tamanho da ninhada e baixa massa relativa da ninhada, semelhante a outras espécies do gênero e espécies unissexuais e bissexuais da família Teiidae.

Palavras-chave: tamanho da ninhada, Ameivula, reprodução, restinga.

\section{REFERENCES}

ANDERSON RA AND VitT LJ. 1990. Sexual selection versus alternative causes of sexual dimorphism in teiid lizards. Oecologia 84(2): 145-157.

AÚN L AND MARTORI R. 1996. Características de la biología de Cnemidophorus serranus y Cnemidophorus lacertoides. Cuad Herpetol 9: 95-99.

BALESTRIN RL, CAPPELlaRi LH AND OUTEIRAL AB. 2010 Biologia reprodutiva de Cercosaura schreibersii (Squamata, Gymnophthalmidae) e Cnemidophorus lacertoides (Squamata, Teiidae) no Escudo Sul-Riograndense, Brasil. Biota Neotrop 10(1): 131-139.

BRANDT R AND NAVAS CA. 2011. Life-History Evolution on Tropidurinae Lizards: Influence of Lineage, Body Size and Climate. Plos One 6(5): e20040.

CAstro F. 1994. Anfibios y Reptiles del Llano. Naturaleza de la Orinoquia. Colombia: Cristina Uribe ediciones, 96 p.

CEI JM AND MARTORI RA. 1991. A New Species of Cnemidophorus of the lacertoides species group from the eastern Sierras de Cordoba, Central Argentina (Lacertilia, Teiidae). Mus Reg Sci Nat Boll (Torino) 9: 33-38.

Cole CJ AND Dessauer HC. 1993. Unisexual and bisexual whiptail lizards of the Cnemidophorus lemniscatus complex (Squamata: Teiidae) of the Guiana Region, South America, with descriptions of new species. Am Mus Novit 3081: 1-30.

COLli GR ET AL. 2003. A new species of Cnemidophorus (Squamata, Teiidae) from the Cerrado biome in central Brazil. Occ Pap Oklahoma Mus Nat Hist 14: 1-14.

Colli GR, Giugliano LG, Mesquita DO AND FranÇA FGR 2009. A new species of Cnemidophorus from the Jalapão region, in the central Brazilian Cerrado. Herpetologica 65(3): 311-327.

CRUZ FB. 1996. Reproductive biology of the lizard Cnemidophorus ocellifer in the dry chaco of Salta, Argentina. Amphibia-Reptilia 17: 80-86.

DEARING MD AND SCHALL JJ. 1994. Atypical reproduction and sexual dimorphism of the tropical Bonaire Island whiptail lizard, Cnemidophorus murinus. Copeia 1994: 760-766. 
DiAs EJR, Rocha CFD AND VRCIBRADIC D. 2002. New Cnemidophorus (Squamata: Teiidae) from Bahia State, northeastern Brazil. Copeia 2002: 928-937.

Dunham AE, MiLEs DB AND RESNICK DN. 1988. Life history patterns in squamate reptiles. In: GANS C AND HUEY RB (Eds), Biology of the Reptilia, Vol. 16, Ecology B, Defense and life history, New York: Alan R. Liss, USA, p. 441-522.

DUMÉRIL AMC AND BIBRON G. 1839. Erpétologie Générale on Histoire Naturelle Complète des Reptiles, Vol. 5, Paris: Roret/Fain et Thunot, $871 \mathrm{p}$.

Feltrim AC AND Lema T. 2000. Uma nova espécie de Cnemidophorus Wagler, 1830 do estado do Rio Grande do sul Brasil (Sauria, Teiidae). Biociências 8(1): 103-114.

Fitch HS. 1981. Sexual size differences in reptiles. Misc Pu Univ Kansas Mus Nat Hist 70: 1-72.

FITCH HS. 1982. Reproductive cycles in tropical reptiles. Misc Pu Univ Kansas Mus Nat Hist 96: 1-53.

FITCH HS. 1985. Variation in Clutch and litter size in New World reptiles. Misc Pu Univ Kansas Mus Nat Hist 76: 1-76.

GALDINO CAB AND VAN SLUYS M. 2011. Clutch size in the small-sized lizard Eurolophosaurus nanuzae (Tropiduridae): Does it vary along the geographic distribution of the species? Iheringia 101(1-2): 61-64.

HaRVEy MB, Ugueto GN AND Gutberlet RL. 2012. Review of teiid morphology with a revised taxonomy and phylogeny of the Teiidae (Lepidosauria: Squamata). Zootaxa 3459: 1-156.

KIEFER M, VAN SLuYs M AND Rocha CFD. 2008. Clutch size and egg size of the tropical lizard Tropidurus torquatus (Tropiduridae) along its geographic range in coastal eastern Brazil. Can J Zool 86: 1376-1388.

KING RB. 2000. Analyzing the relationship between clutch size and female body size in reptiles. J Herpetol 34: 148-150.

LAURENTI JN. 1768. Specimen medicum, exhibens synopsin reptilium emendatam cum experimentis circa venena et antidota reptilium austracorum, quod authoritate et consenso, Vienna: Joan. Thomae, $217 \mathrm{p}$.

LEÓN JR AND CovA LJ. 1973. Reproducción de Cnemidophorus lemniscatus (Sauria: Teiidae) en Cumaná, Venezuela. Caribb J Sci 13: 63-68.

MAGNUSSON WE. 1987. Reproductive cycles of teiid lizards in Amazonian Savanna. J Herpetol 21: 307-316.

MARKEZICH AL, COLE CJ AND DESSAUER HC. 1997. The blue and green whiptail lizards (Squamata: Teiidae: Cnemidophorus) of the Peninsula de Paraguana, Venezuela: Systematics, ecology, descriptions of two new taxa, and relationships to whiptails of the Guianas. Am Mus Nov 3207: 1-60.

MCCRYSTAL HK AND DIXON JR. 1987. A new species of Cnemidophorus (Sauria Teiidae) from the llanos of Colombia and Venezuela. J Herpetol 21: 245-254.

MENEZES VA AND RocHA CFD. 2013. Geographic distribution, population densities, and issues on conservation of whiptail lizards in restinga habitats along the eastern coast of Brazil. N West J Zool 2(9): 337-344.
Menezes VA, Rocha CFD AND DUTRA GF. 2004. Reproductive ecology of the parthenogenetic whiptail lizard Cnemidophorus nativo in a Brazilian restinga habitat. J Herpetol 38(2): 280-282.

Menezes VA, VAN SLuYs M, Fontes AF AND Rocha CFD. 2011. Living in a caatinga-rocky field transitional habitat: ecological aspects of the whiptail lizard Cnemidophorus ocellifer (Teiidae) in northeastern Brazil. Zool 28: 8-16.

Mesquita DO AND COLLI GR. 2003a. Geographical variation in the ecology of populations of some Brazilian species of Cnemidophorus (Squamata, Teiidae). Copeia 2003(2): 305-329.

Mesquita DO AND Colli GR. 2003b. The ecology of Cnemidophorus ocellifer (Squamata, Teiidae) in a Neotropical Savanna. J Herpetol 37(3): 498-509.

MoJICA BH, Rey BH, SERRANO VH AND RAMIREZ-PINILLA MP. 2003. Annual reproductive activity of a population of Cnemidophorus lemniscatus (Squamata: Teiidae). J Herpetol 37(1): 35-42.

MUEHE D. 1983. Conseqüências higroclimáticas das glaciações quartenárias no relevo costeiro a leste da Baía de Guanabara. Rev Brasil Geociên 13: 245-252.

NIEWIAROWSKI PH. 1994. Understanding geographic lifehistory variation in lizards. In: VITT LJ and PIANKA ER (Eds), Lizard ecology: historical and experimental perspectives. New Jersey: Princeton University, p. 31-49.

PERRIN P. 1984. Evolução da costa fluminense entre as pontas de Itacoatiara e negra: Preenchimentos e restingas. In: LACERDA LD ET AL. (Eds), Restingas: origem, estrutura, processos. Niterói: CEUFF, Brazil, p. 65-74.

RAMÍREZ-BAUTISTA A, BALDERAS-VALDIVIA C AND VitT LJ. 2000. Reproductive ecology of the whiptail lizard Cnemidophorus lineatissimus (Squamata: Teiidae) in a tropical dry forest. Copeia 2000: 712-722.

RAMÍREZ-BAUTISTA A AND VITT LJ. 1998. Reproductive biology of Urosaurus bicarinatus (Sauria: Phrynosomatidae) from a tropical dry forest of México. Southwest Nat 43: 381-390.

ReEder TW, Cole CJ AND Dessauer HC. 2002. Phylogenetic relationships of whiptail lizards of the genus Cnemidophorus (Squamata: Teiidae): a test of monophyly, reevaluation of karyotypic evolution, and review of hybrid origins. Am Mus Novit 3365: 1-61.

REZENDE-PINTO FM, VERRASTRO L, ZANOTELLI JC AND BARATA PCR. 2009. Reproductive biology and sexual dimorphism in Cnemidophorus vacariensis (Sauria, Teiidae) in the grasslands of the Araucaria Plateau, southern Brazil. Iheringia Ser Zool 99:82-91.

RocHA CFD. 1992. Reproductive and fat body cycles of the tropical sand lizard (Liolaemus lutzae) of southeastern Brazil. J Herpetol 26: 17-23.

RochA CFD. 2008. Body size, female reproduction and sexual dimorphism in the lizard Ameiva ameiva (Teiidae) in a restinga of southeastern Brazil. Rev Bras Zool 25: 370-372.

Rocha CFD, Bergallo HG and Peccinini-Seale D. 1997. Evidence of an unisexual population of the brazilian whiptail lizard genus Cemidophorus (Teiidae), with description of a new species. Herpetologica 53: 374-382. 
Rocha CFD, Menezes VA, Bergallo HG AND Dutra GF. 1999. Cnemidophorus nativo Geographic Distribution. Herpetol Rev 30: 109.

RODRIGUEZ-RAMIREZ J AND LEWIS AR. 1991. Reproduction in the Puerto Rican teiids Ameiva exsul and A. wetmorei. Herpetologica 47: 395-403.

SANTANA GG, VAsconcellos A, Gadelha YEA, Vieira WLS, ALMEIDA WO, NÓBREGA RP AND ALVES RRN. 2010. Feeding habits, sexual dimorphism and size at maturity of the lizard Cnemidophorus ocellifer (Spix, 1825) (Teiidae) in a reforested restinga habitat in northeastern Brazil. Braz J Biol 70(2): 409-416.

SCHALL JJ. 1978. Reproductive Strategies in Sympatric Whiptail Lizards (Cnemidophorus): Two Parthenogenetic and Three Bisexual Species. Copeia 1978(1): 108-116.

SCHALL JJ. 1983. Small Clutch Size in a Tropical Whiptail Lizard (Cnemidophorus arubensis). J Herpetol 17(4): 406-408.

SHINE R. 1980. "Costs" of reproduction in reptiles. Oecologia 46: $92-100$

SHINE R AND ELPHICK MJ. 2001. The effect of short-term weather fluctuations on temperatures inside lizard nests, and on the phenotypic traits of hatchling lizards. Biol J Linn Soc 72: 555-565.

SHINE R AND GEER AE. 1991. Why are clutch sizes more variable in some species than in others? Evolution 45: 1696-1706

Sinervo B, Doughty P, Huey HP AND Zamudio K. 1992 Allometric engineering: a causal analysis of natural selection on offspring size. Science 258: 1927-1930.

Suguio K AND TeSsler MG. 1984. Planícies de cordões arenosos Quaternários do Brasil: origem e nomenclatura. In: LACERDA LD ET AL. (Eds), Restingas: Origem, Estrutura, Processos. Niterói: CEUFF, Brazil, p. 15-26.

VAN LIDTH DE JEUDE THW. 1887. On a collection of reptiles and fishes from the West-Indies. Notes from the Leyden Museum 9: 129-139.

VAN SLuYs M. 1998. Growth and Body Condition of the Saxicolous Lizard Tropidurus itambere in Southeastern Brazil. J Herpetol 32: 359-365.

VAn Sluys M, Martelotte SB, KiefFer MC and Rocha CFD. 2010. Reproduction in neotropical Tropidurus lizards (Tropiduridae): evaluating the effect of environmental factors on T. torquatus. Amphibia-Reptilia 31: 117-126.
VANZOLINI PE. 1970. Unisexual Cnemidophorus lemniscatus in the Amazonas valley: A preliminary note (Sauria, Teiidae). Pap Avulsos Zool 23: 63-68.

VITT LJ. 1983. Reproduction and sexual dimorphism in the tropical teiid lizard Cnemidophorus ocellifer. Copeia 1983: 359-366.

VITT LJ. 1991. An introduction of the ecology of cerrado lizards. J Herpetol 25(1): 79-90.

VitT LJ AND BREITENBACH GL. 1993. Life histories and reproductive tactics among lizards in the genus Cnemidophorus (Sauria: Teiidae). In: WRIGHT JW AND VITT LJ (Eds), Biology of whiptail lizards (Genus Cnemidophorus), Oklahoma: The Oklahoma Museum of Natural History, USA, p. 211-243.

ViTT LJ AND CONGDON JD. 1978. Body shape, reproductive effort, and relative clutch mass in lizards: resolution of a paradox. Am Nat 112: 595-608.

VITT LJ AND COOPER WE. 1985. The relationship between reproduction and lipid cycling in the skink Eumeces laticeps with comments on brooding ecology. Herpetologica 41(4): 419-432.

VitT LJ AND PRICE HJ. 1982. Ecological and evolutionary determinants of relative clutch mass in lizards. Herpetologica 38: 237-255.

VitT LJ, ZANI PA, CALDWELl JP, ARAÚJO MC AND MAGNUSSON WE. 1997. Ecology of whiptail lizards (Cnemidophorus) in the Amazon region of Brazil. Copeia 1997: 745-757.

VRCIBRADIC D AND ROCHA CFD. 2011. An overview of female reproductive traits in South American Mabuyaa (Squamata, Scincidae), with emphasis on brood size and its correlates. J Nat Hist 45: 13-14.

WINCK GR AND Rocha CFD. 2012. Reproductive Trends of Brazilian Lizards (Reptilia, Squamata): The Relationship between Clutch Size and Body Size in Females. N West J Zool: 8: 57-62.

ZAR JH. 1999. Biostatistical Analysis. $4^{\text {th }}$ ed., Prentice Hall Inc., New Jersey: Upper Saddle River, USA, 662 p. 